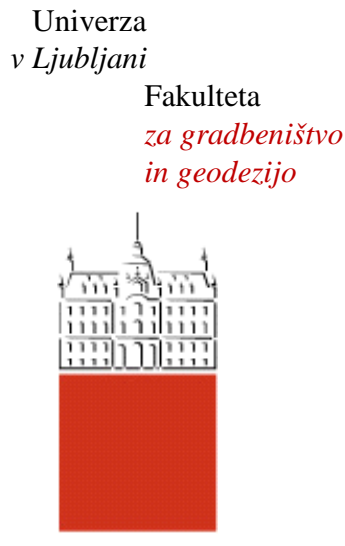

Jamova 2

1000 Ljubljana, Slovenija http://www3.fgg.uni-lj.si/

DRUGG - Digitalni repozitorij UL FGG http://drugg.fgg.uni-lj.si/

Ta članek je avtorjeva zadnja recenzirana različica, kot je bila sprejeta po opravljeni recenziji.

Prosimo, da se pri navajanju sklicujete na bibliografske podatke, kot je navedeno:

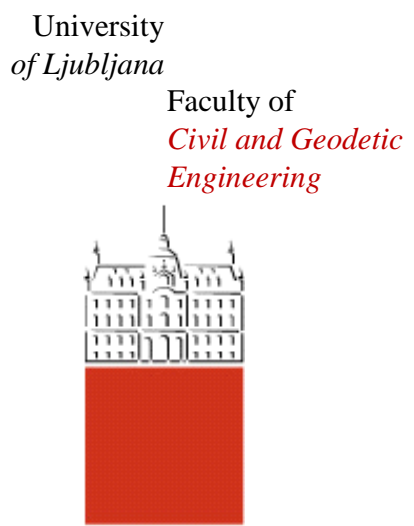

Jamova 2

SI - 1000 Ljubljana, Slovenia http://www3.fgg.uni-lj.si/en/

DRUGG - The Digital Repository http://drugg.fgg.uni-lj.si/

This version of the article is author's manuscript as accepted for publishing after the review process.

When citing, please refer to the publisher's bibliographic information as follows: 


\title{
A KINEMATICALLY EXACT FINITE ELEMENT FORMULATION OF ELASTIC-PLASTIC CURVED BEAMS
}

\author{
M. SAJE*, G. TURK, A. KALAGASIDU and B. VRATANAR \\ University of Ljubljana, Faculty of Civil and Geodetic Engineering, \\ Jamova 2, SI-1001 Ljubljana, Slovenia, P.O. Box 579
}

\begin{abstract}
A finite element, large displacement formulation of static elasticplastic analysis of slender arbitrarily curved planar beams is presented. Nonconservative and dynamic loads are at present not included. The Bernoulli hypothesis of plane cross-sections is assumed and the effect of shear strains is neglected. Exact nonlinear kinematic equations of curved beams derived by Reissner [J. appl. Math. Phys. (ZAMP) 23, 795-804 (1972)] are incorporated into a generalized principle of virtual work through Lagrangian multipliers. The only function that has to be interpolated in the finite element implementation is the rotation of the centroid axis of a beam. This is an important advantage over other classical displacement approaches since the field consistency problem and related locking phenomena do not arise. Numerical examples, comprising elastic and elastic-plastic, curved and straight beams, at large displacements and rotations, show very nice computational and accuracy characteristics of the present family of finite elements. The comparisons to other published results very clearly show the superior performance of the present elements.
\end{abstract}

\section{INTRODUCTION}

A family of new slender beam finite elements with arbitrarily curved centroid axis for the kinematically exact static analysis of elastic-plastic planar frames based on assumed rotation variation is presented here. Reissner's nonlinear beam theory [28] is the basis of our finite

${ }^{*}$ Corresponding author (email: msaje@fagg.uni-lj.si) 
element formulation. There are no mathematical restrictions regarding the magnitude of displacements, rotations, and strains. The effect of shear strain is at present not included.

Only for some structures of specific geometrical and loading data, and elastic material analytical solutions exist in terms of elliptic integrals or infinite series. Such simple examples of elastic frames are presented in, e.g., [9], [13] and [34]. In a more general context, numerical methods have to be employed. In the present paper, alike in most publications, the finite element method is used.

A majority of publications devoted to curved beams is limited to geometrically and materially linear analyses. In this context, considerable attention has been focused to alleviate the shear and membrane locking in displacement based curved beam formulations, and a number of different approaches have been proposed. These are the strain element technique [5], reduced integration [23], [38], [39], hybrid/mixed formulation [27], [32], mixed formulation in conjunction with collocation [26], shear penalty-parameter modifications [42], field consistency [1], [19], a hierarchical displacement interpolation concept [15], a separation of deformation mode for radial displacement [21], and others [47]. Only some of these locking cures can be applied in the geometrically nonlinear curved beam analysis [16].

The present finite element curved beam formulation may be roughly classified as the one based on assumed strains. For geometrically nonlinear case and elastic material, its implementation was described in [29] for straight and in [30] for curved beams. Only the rotation function has to be approximated in these formulations. Because the curvature is the derivative of the rotation with respect to the axis coordinate, this is essentially equal to assuming only the variation of the curvature. The remaining two deformation quantities, i.e., extensional and shear strains, are obtained from constitutive and equilibrium equations. Furthermore, because the problem is stated in a variational form of the Hu-Washizu functional, the variation of displacements along the beam axis is not needed and thus no approximation of displacements is required. A similar concept of basing the formulation on curvature approximation but otherwise employing standard displacement approach, was later presented by Lee and Sin [20] in a narrower framework of geometrically and materially linear curved beam analysis. A pure strain assumed finite element formulation of linear curved beams was proposed by Choi and Lim [5]. Their formulation indeed alleviate locking and is rather accurate, but assumes more functions to be approximated than required, i.e., extensional and shear strains in addition to the curvature, and is therefore computationally less efficient compared to [20]. The approach in [20] may be considered as being a linearized version of the formulation presented 
in [30].

For geometrically nonlinear curved beams a number of finite element formulations have been developed recently. Some propose a corotational, kinematically "inexact" formulation, e.g. Hsiao and Hou [14], using assumptions of small deflection beam theory and constant extensional strain. Sandhu et al. [33] develop another very efficient and reliable corotational formulation (see also [10], [24] and [46]). Moreover, a kinematically exact formulations have also been proposed. For instance, Surana [40], [41] presents the formulation in which the element displacement field approximation is expressed in terms of nodal translations and nonlinear functions of nodal rotations. Ibrahimbegović and Frey [16] discuss the locking cures in geometrically nonlinear analysis of planar analysis of shear flexible Reissner's beam theory. Pak and Stauffer [25] reduce equations of curved elastic beam to a single second-order nonlinear ordinary differential equation and a resulting two-point boundary value problem is solved by a finite element method. As in [30] only the rotation function needs to be interpolated. Kegl et al. [18], on the basis of Saje's exact kinematics element [30], propose a modified formulation of this element for shape optimal design, employing the Bezier curves. The Bezier curves concept is also used in a recent paper by Gontier and Vollmer [12], where Simo's [36] geometrically exact formulation is adapted. Three-dimensional arbitrary curved beam elements employing Reissner's beam theory are derived by Ibrahimbegović [15]. His finite elements assume displacement and rotation interpolations, formulate the element matrices directly in the global coordinate system, and are free from locking. A finite volumes method in conjunction with a mixed form variational principle is used by Franchi and Montelaghi [11]. This approach is attractive because it allows to use only one node inside the element and the weakest continuity for test and trial functions. Locking phenomenon is intrinsically avoided in their elements.

The extension of an elasticity-based formulation to the analysis of an elastic-plastic beam is by no means trivial. The literature combining plasticity, curved beams and exact geometrical nonlinearity is scarce. An excellent elastic-visco-plastic formulation employing exact kinematics, yet for straight beams, is given by Simo et al. [35]. A comprehensive list of other references on straight beam elastic-plastic, large displacement finite element formulations is given in [31]. Neither of those, however, employs exact kinematical relationships.

The present paper is an extension of two our previous papers: on nonlinear behavior of straight slender elastic-plastic planar beams [31], and on nonlinear behavior of curved elastic beams [30]. The nonlinear, geometrically exact elastic analysis of the curved beam presented 
in [30] is extended to include a general nonlinear material. The formulation can account for a variety of nonlinear material models, but an elastic-plastic material law is presented here for the sake of concretness. As in [30] and [31], the only unknown function that has to be interpolated over the finite element is the rotation of the centroid axis of the beam. This feature inherently avoids locking, and assures high accuracy and computational efficiency, which is demonstrated by several numerical examples presented at the end of the paper. An important difference from the formulation in [31] is that the formulation is given directly in the global coordinate system of a structure, so that no local-global transformations of element arrays needs to be done. The effect of shear strains is neglected but can be accounted for easily along the lines given in [30].

\section{KINEMATIC RELATIONS. CONSTITUTIVE EQUATIONS.}

An undeformed beam is described by its centroid axis which is an arbitrary smooth curve in the $(x, z)$ plane of the fixed-in-space coordinate system with the unit base vectors $\mathbf{e}_{x}, \mathbf{e}_{y}$ and $\mathbf{e}_{z}$. The centroid axis of the undeformed beam is defined by the parametric representation $(x(s), z(s))$, where $s \in[0, L]$ is the curvilinear coordinate (the arc length) spanning the axis of the undeformed beam of length $L$. The initial curvature of the beam, $\varphi_{0}^{\prime}(s)$, is an arbitrary function of $s$, too. The cross-section, $\mathcal{A}$, is for simplicity assumed to be constant along the beam, and to lay in a plane perpendicular to the centroid axis. A material point of the beam, $\mathcal{P}$, is represented by material coordinates $(\xi, \eta, \zeta)$, where $\xi$ coincides with $s$, coordinate $\eta$ coincides with $y$, and $\zeta$ is perpendicular to axes $\xi$ and $\eta$ (see Fig. 1). It is furthermore assumed that the cross-section is symmetric with respect to axis $\zeta$. Thus, the variables used in the formulation are not functions of coordinate $y=\eta$.

According to Reissner's beam theory [28], components $u(s)$ and $w(s)$ of the displacement vector $\mathbf{u}(s)$ of the centroid axis

$$
\mathbf{u}(s)=u(s) \mathbf{e}_{x}+w(s) \mathbf{e}_{z}
$$

are related to extensional strain of the centroid axis, $\varepsilon(s)$, and the rotation of the centroid axis relative to $x$-axis, $\varphi(s)$, by equations

$$
\begin{aligned}
& x^{\prime}+u^{\prime}-(1+\varepsilon) \cos \varphi=0, \\
& z^{\prime}+w^{\prime}+(1+\varepsilon) \sin \varphi=0 .
\end{aligned}
$$


Prime $(')$ denotes the derivative with respect to $s$. For a slender curved beam, extensional strain $D(s, \zeta)$ of an arbitrary longitudinal fibre is taken to be

$$
D(s, \zeta)=\varepsilon(s)+\zeta \Delta \varphi^{\prime}(s)
$$

where $\Delta \varphi^{\prime}(s)$, the change of curvature due to deformations, is given by

$$
\Delta \varphi^{\prime}(s)=\varphi^{\prime}(s)-\varphi_{0}^{\prime}(s)
$$

$\varphi_{0}(s)$ is the rotation of the undeformed centroid axis with respect to $x$-axis (Fig. 1). In theoretical considerations, a nonlinear material model of the form $\sigma=f(D)$ is taken into account. A bilinear elastic-plastic material model is used in numerical examples, and defined by the equation

$$
\sigma=\left\{\begin{array}{ll}
E D & \text { for }|D| \leq D_{Y} \\
E D_{Y} \operatorname{sgn} D+E_{p}\left(D-D_{Y} \operatorname{sgn} D\right) & \text { for }|D|>D_{Y}
\end{array},\right.
$$

where $E, E_{p}$, and $D_{Y}$ are elastic modulus, plastic modulus, and extensional yield strain, respectively. The unloading is considered via kinematic or isotropic hardening rules.

\section{PRINCIPLE OF VIRTUAL WORK}

The principle of virtual work for a curved beam element of initial length $L$ and initial curvature $\varphi_{0}^{\prime}(s)$, subjected to distributed loads $p_{x}, p_{z}$, and $m_{y}$, and end-point loads $S_{k}(k=$ $1,2, \ldots, 6)$ states $[44]$

$$
\int_{0}^{L} \int_{\mathcal{A}} \sigma \delta D d A d s=\int_{0}^{L} p_{x} \delta u d s+\int_{0}^{L} p_{z} \delta w d s+\int_{0}^{L} m_{y} \delta \varphi d s+\sum_{k=1}^{6} S_{k} \delta U_{k},
$$

where $\delta D, \delta u, \delta w, \delta \varphi$, and $\delta U_{k}$ denote virtual extensional strain, virtual displacements in $x$ and $z$ directions, a virtual rotation, and virtual displacements of end points, respectively. A virtual extensional strain is related to virtual deformation quantities $\delta \varepsilon$ and $\delta \varphi$ by the equation

$$
\delta D=\delta \varepsilon+\zeta \delta \varphi^{\prime}
$$

obtained by varying eqns (4) and (5). After eqn (8) has been inserted in (7), the principle of virtual work reads

$$
\int_{0}^{L}\left(\mathcal{N}_{c} \delta \varepsilon+\mathcal{M}_{c} \delta \varphi^{\prime}\right) d s-\int_{0}^{L} p_{x} \delta u d s-\int_{0}^{L} p_{z} \delta w d s-\int_{0}^{L} m_{y} \delta \varphi d s-\sum_{k=1}^{6} S_{k} \delta U_{k}=0
$$


By $\mathcal{N}_{c}(s)$ and $\mathcal{M}_{c}(s)$ we denote the cross-sectional stress-resultants (the axial force and the bending moment) defined by equations

$$
\begin{aligned}
& \mathcal{N}_{c}(s)=\int_{\mathcal{A}} \sigma(s, \zeta) d A, \\
& \mathcal{M}_{c}(s)=\int_{\mathcal{A}} \zeta \sigma(s, \zeta) d A .
\end{aligned}
$$

In order to account for exact kinematic eqns (2) and (3) the generalized principle of virtual work is employed by introducing eqns (2) and (3) into the principle of virtual work through Lagrangian multipliers $\lambda_{1}(s)$ and $\lambda_{2}(s)$. Equations

$$
\begin{aligned}
& \int_{0}^{L}\left[x^{\prime}+u^{\prime}-(1+\varepsilon) \cos \varphi\right] \lambda_{1} d s=0, \\
& \int_{0}^{L}\left[z^{\prime}+w^{\prime}+(1+\varepsilon) \sin \varphi\right] \lambda_{2} d s=0
\end{aligned}
$$

are varied with respect to $u, w, \varepsilon, \varphi, \lambda_{1}$, and $\lambda_{2}$, and the resulting equations (see [31] for more details) are added to the principle of virtual work, eqn (9). After the terms $\mathcal{M}_{c} \delta \varphi^{\prime}, \lambda_{1} \delta u^{\prime}$, and $\lambda_{2} \delta w^{\prime}$ are integrated by parts, the extended eqn (9) yields

$$
\begin{aligned}
0 & =\int_{0}^{L}\left[\mathcal{N}_{c}-\lambda_{1} \cos \varphi+\lambda_{2} \sin \varphi\right] \delta \varepsilon d s+ \\
& +\int_{0}^{L}\left[-\mathcal{M}_{c}^{\prime}+(1+\varepsilon)\left(\lambda_{1} \sin \varphi+\lambda_{2} \cos \varphi\right)-m_{y}\right] \delta \varphi d s+ \\
& +\int_{0}^{L}\left(-p_{x}-\lambda_{1}^{\prime}\right) \delta u d s+\int_{0}^{L}\left(-p_{z}-\lambda_{2}^{\prime}\right) \delta w d s+ \\
& +\int_{0}^{L}\left[x^{\prime}+u^{\prime}-(1+\varepsilon) \cos \varphi\right] \delta \lambda_{1} d s+ \\
& +\int_{0}^{L}\left[z^{\prime}+w^{\prime}+(1+\varepsilon) \sin \varphi\right] \delta \lambda_{2} d s- \\
& -\sum_{k=1}^{6} S_{k} \delta U_{k}+\lambda_{1}(L) \delta u(L)-\lambda_{1}(0) \delta u(0)+\lambda_{2}(L) \delta w(L)- \\
& -\lambda_{2}(0) \delta w(0)+\mathcal{M}_{c}(L) \delta \varphi(L)-\mathcal{M}_{c}(0) \delta \varphi(0) .
\end{aligned}
$$

Here, variations $\delta u, \delta w, \delta \varepsilon, \delta \varphi, \delta \lambda_{1}$, and $\delta \lambda_{2}$ are arbitrary independent functions, while variations $\delta U_{1}=\delta u(0), \delta U_{2}=\delta w(0), \delta U_{3}=\delta \varphi(0), \delta U_{4}=\delta u(L), \delta U_{5}=\delta w(L)$, and $\delta U_{6}=$ $\delta \varphi(L)$ are arbitrary independent parameters. Therefore, the coefficients at the variations should be zero for eqn (13) to be satisfied, which yields the following set of equilibrium and kinematic equations, and the related natural boundary conditions:

$$
\begin{aligned}
x \in[0, L]: \quad \delta \varepsilon & \rightarrow \mathcal{N}_{c}-\lambda_{1} \cos \varphi+\lambda_{2} \sin \varphi=0, \\
x \in(0, L): \delta \varphi & \rightarrow-\mathcal{M}_{c}^{\prime}+\left(\lambda_{1} \sin \varphi+\lambda_{2} \cos \varphi\right)(1+\varepsilon)-m_{y}=0, \\
\delta u & \rightarrow \lambda_{1}^{\prime}+p_{x}=0
\end{aligned}
$$


$\delta w \quad \rightarrow \quad \lambda_{2}^{\prime}+p_{z}=0$

$\delta \lambda_{1} \rightarrow x^{\prime}+u^{\prime}-(1+\varepsilon) \cos \varphi=0$,

$\delta \lambda_{2} \quad \rightarrow \quad z^{\prime}+w^{\prime}+(1+\varepsilon) \sin \varphi=0$,

$x=0: \delta U_{1} \quad \rightarrow \quad S_{1}+\lambda_{1}(0)=0$,

$\delta U_{2} \rightarrow S_{2}+\lambda_{2}(0)=0$,

$\delta U_{3} \quad \rightarrow \quad S_{3}+\mathcal{M}_{c}(0)=0$,

$x=L: \delta U_{4} \quad \rightarrow \quad S_{4}-\lambda_{1}(L)=0$,

$\delta U_{5} \quad \rightarrow \quad S_{5}-\lambda_{2}(L)=0$,

$\delta U_{6} \quad \rightarrow \quad S_{6}-\mathcal{M}_{c}(L)=0$.

Eqns (14)-(19) build up a system of six algebraic-differential equations for six unknown functions $\lambda_{1}(s), \lambda_{2}(s), \varphi(s), \varepsilon(s), u(s)$, and $w(s)$ with the related boundary conditions, eqns (20)-(25). Fortunately, all the equations are not coupled. Eqns (14), (16), and (17) can be solved separately. In fact, eqns (16) and (17) may easily be integrated to obtain

$$
\begin{array}{ll}
\lambda_{1}(s)=\lambda_{1}^{0}-\int_{0}^{s} p_{x}(\xi) d \xi, & \lambda_{1}^{0}=\lambda_{1}(0), \\
\lambda_{2}(s)=\lambda_{2}^{0}-\int_{0}^{s} p_{z}(\xi) d \xi, & \lambda_{2}^{0}=\lambda_{2}(0) .
\end{array}
$$

Eqn (14) furnishes the value of extensional strain $\varepsilon$ as a function of $\varphi, \lambda_{1}^{0}$, and $\lambda_{2}^{0}$, which can be, for a chosen nonlinear constitutive equation, obtained numerically. Once eqns (14), (16), and (17) are satisfied, the first, third, and fourth integral in eqn (13) vanish. After partially integrating the terms $u^{\prime} \delta \lambda_{1}, w^{\prime} \delta \lambda_{2}, \mathcal{M}_{c}^{\prime} \delta \varphi$, and considering that $\delta \lambda_{1}(s)=\delta \lambda_{1}^{0}$ and $\delta \lambda_{2}(s)=\delta \lambda_{2}^{0}$, the generalized principle of virtual work (13) may be rewritten as

$$
\begin{aligned}
0 & =\left[-\int_{0}^{L}(1+\varepsilon) \cos \varphi d s+x(L)-x(0)+U_{4}-U_{1}\right] \delta \lambda_{1}^{0}+ \\
& +\left[\int_{0}^{L}(1+\varepsilon) \sin \varphi d s+z(L)-z(0)+U_{5}-U_{2}\right] \delta \lambda_{2}^{0}+ \\
& +\int_{0}^{L}\left\{\mathcal{M}_{c} \delta \varphi^{\prime}+\left[(1+\varepsilon)\left(\lambda_{1} \sin \varphi+\lambda_{2} \cos \varphi\right)-m_{y}\right] \delta \varphi\right\} d s- \\
& -\left(S_{1}+\lambda_{1}^{0}\right) \delta U_{1}-\left(S_{2}+\lambda_{2}^{0}\right) \delta U_{2}-S_{3} \delta U_{3}- \\
& -\left[S_{4}-\lambda_{1}(L)\right] \delta U_{4}-\left[S_{5}-\lambda_{2}(L)\right] \delta U_{5}-S_{6} \delta U_{6} .
\end{aligned}
$$

As a result, there is only one unknown function - rotation $\varphi(s)$ in eqn $(27)$ which needs to be approximated in the finite element implementation. This is an important advantage compared to those formulations where more than one function have to be approximated. 
Hence the problem of inconsistent field interpolations does not arise and membrane locking phenomenon is inherently avoided.

\section{FINITE ELEMENT FORMULATION}

Using $M$ equidistant points, the finite element is divided into $M-1$ sections (Fig. 2). Therefore, rotation $\varphi(s)$ is approximated by a set of Lagrangian polynomials $I_{i}(s)(i=$ $1,2, \ldots, M)$ of order $(M-1)$ by the expression

$$
\varphi(s)=\sum_{i=1}^{M} I_{i}(s) \varphi_{i}
$$

where $\varphi_{i}(i=1,2, \ldots, M)$ are nodal points rotations. From eqn (28) it follows

$$
\varphi^{\prime}(s)=\sum_{i=1}^{M} I_{i}^{\prime}(s) \varphi_{i}, \quad \delta \varphi(s)=\sum_{i=1}^{M} I_{i}(s) \delta \varphi_{i}, \quad \delta \varphi^{\prime}(s)=\sum_{i=1}^{M} I_{i}^{\prime}(s) \delta \varphi_{i} .
$$

By using eqns (28) and (29) in eqn (27), and by zeroing factors of independent variations $\delta \lambda_{1}^{0}$, $\delta \lambda_{2}^{0}, \delta \varphi_{i}(i=1,2, \ldots, M), \delta U_{1}, \delta U_{2}, \delta U_{4}$, and $\delta U_{5}$, we obtain the system of $M+6$ nonlinear equations for $M+6$ unknown parameters $\lambda_{1}^{0}, \lambda_{2}^{0}, \varphi_{i}(i=1,2, \ldots, M), U_{1}, U_{2}, U_{4}$, and $U_{5}$ :

$$
\begin{aligned}
& f_{1}\left(\lambda_{1}^{0}, \lambda_{2}^{0}, \varphi_{i}, U_{1}, U_{4}\right)=-\int_{0}^{L}(1+\varepsilon) \cos \varphi d s+x(L)-x(0)+U_{4}-U_{1}=0, \\
& f_{2}\left(\lambda_{1}^{0}, \lambda_{2}^{0}, \varphi_{i}, U_{2}, U_{5}\right)=\int_{0}^{L}(1+\varepsilon) \sin \varphi d s+z(L)-z(0)+U_{5}-U_{2}=0, \\
& f_{2+i}\left(\lambda_{1}^{0}, \lambda_{2}^{0}, \varphi_{i}\right)=\int_{0}^{L}\left\{\mathcal{M}_{c} I_{i}^{\prime}+\left[(1+\varepsilon)\left(\lambda_{1} \sin \varphi+\lambda_{2} \cos \varphi\right)-m_{y}\right] I_{i}\right\} d s-S_{i}^{*}=0, \\
& \quad(i=1,2, \ldots, M), \\
& f_{2+M+1}\left(\lambda_{1}^{0}\right)=-\left(S_{1}+\lambda_{1}^{0}\right)=0, \\
& f_{2+M+2}\left(\lambda_{2}^{0}\right)=-\left(S_{2}+\lambda_{2}^{0}\right)=0, \\
& f_{2+M+3}\left(\lambda_{1}^{0}\right)=-\left(S_{4}-\lambda_{1}(L)\right)=0, \\
& f_{2+M+4}\left(\lambda_{2}^{0}\right)=-\left(S_{5}-\lambda_{2}(L)\right)=0 .
\end{aligned}
$$

In eqn (32), $S_{i}^{*}$ are defined as follows: $S_{1}^{*}=S_{3}, S_{M}^{*}=S_{6}, S_{i}^{*}=0$ for $i=2,3, \ldots, M-1$.

The system of nonlinear eqns (30)-(36) is solved by Newton's iteration method. In the iteration step $k+1(k=0,1,2, \ldots)$, eqns (30)-(36) are linearized yielding a symmetric system of $M+6$ linear equations for unknown increments $\Delta \lambda_{1}^{0}, \Delta \lambda_{2}^{0}, \Delta \varphi_{i}(i=1,2, \ldots, M), \Delta U_{1}$, $\Delta U_{2}, \Delta U_{4}$, and $\Delta U_{5}$ : 


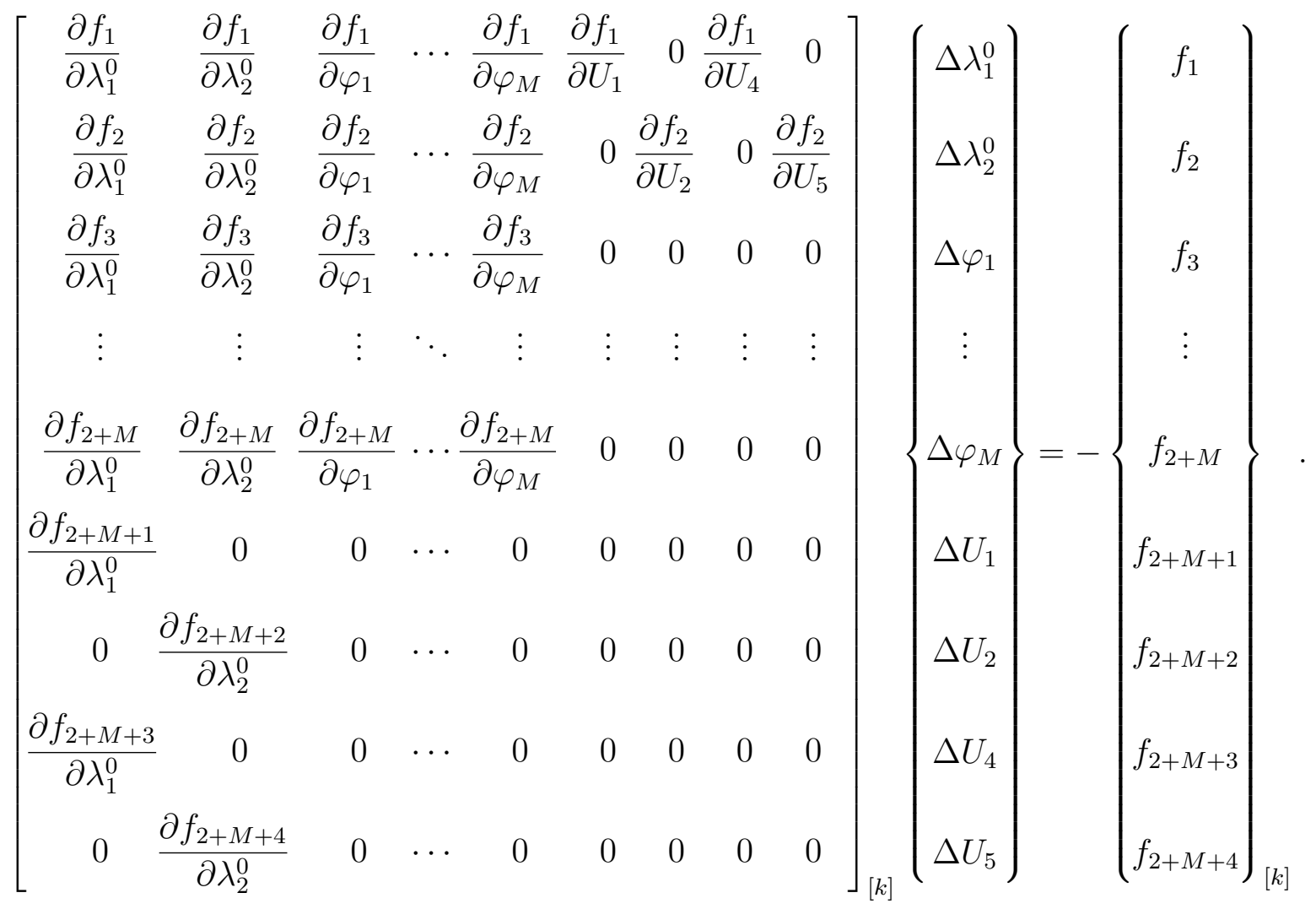

Subscript $[k]$ indicates that a term is evaluated using values obtained at the $k$-th iteration step. From $M+6$ unknown parameters only six are identified as external: $\Delta U_{1}, \Delta U_{2}, \Delta U_{3}=\Delta \varphi_{1}$, $\Delta U_{4}, \Delta U_{5}$, and $\Delta U_{6}=\Delta \varphi_{M}$. Others are considered as being internal degrees of freedom and are eliminated on the element level by a standard condensation algorithm. In the iteration step $k$, the condensed element tangent stiffness matrix, $\mathbf{K}^{[k]}$, and the associated unbalanced nodal force vector, $\mathbf{F}^{[k]}$, are thus related by a linear equation

$$
\mathbf{K}^{[k]} \boldsymbol{\Delta} \mathbf{U}^{[k]}=\mathbf{F}^{[k]},
$$

where $\Delta \mathbf{U}^{[k]}=\left\langle\Delta U_{1}, \Delta U_{2}, \Delta U_{3}, \Delta U_{4}, \Delta U_{5}, \Delta U_{6}\right\rangle$ is the vector of external degrees of freedom. Improved values of the unknowns are obtained from the equation

$$
\mathbf{U}^{[k+1]}=\mathbf{U}^{[k]}+\Delta \mathbf{U}^{[k]}, \quad k=0,1,2, \ldots
$$

The system of eqns (38) corresponds to the global coordinate system $(x, z)$ of a structure, meaning, that, within the present formulation, there is no need of the coordinate transformation.

The determination of the tangent stiffness matrix and the unbalanced load vector is explained in detail in [31]. The explicit expressions of the coefficients of the system matrix of eqn (37) are given in Appendix. As observed in Appendix, integrations along the beam axis 
(eqns $(A)$ of Appendix), and integrations over the cross-section (i.e., cross-sectional stiffnesses $C_{11}, C_{12}$, and $C_{22}$, see eqns $(B)$ of Appendix), are required.

In some specific examples it may indeed be possible to evaluate integrals over the crosssection analytically. For the linear elastic material law this evaluation is straight-forward. If the bilinear hyper-elastic type of elastic-plastic law is employed, analytical evaluation can also be easily performed since the loading history is not important. Otherwise the numerical integration is needed. Lobatto's or Gaussian integration rules are usually used. Cross-sectional integrals $C_{11}, C_{12}$, and $C_{22}$ (see eqns $(B)$ of Appendix) assume the integration of the uniaxial tangent modulus of material, $E_{t}=\partial \sigma / \partial D$, over the cross-section. Since $E_{t}$ is generally not continuous function over the cross-section, the numerical integration may lead to rather inaccurate results, as it has been shown in [31] by a means of a simple example. The inaccuracies may be significant and an increased number of integration points may, somewhat surprisingly, lead to even worse results. This will further be commented upon in numerical examples. Note, however, that the same problem of integrating discontinuous functions to obtain cross-sectional stiffnesses arises in any elastic-plastic beam, plate or shell theory. An extensive overview of numerical integration strategies is given in [4], but an optimal method has still to be derived.

Because trigonometric functions appear in eqns $(A)$ of Appendix, the integration along the element axis can not be performed analytically. Therefore, the numerical integration must be used. Moreover, in a partially plastified beam element for bilinear elastic-plastic material model, cross-sectional stiffnesses $C_{11}, C_{12}$, and $C_{22}$ are no longer differentiable functions of $s$ (though they are still continuous; see numerical example 6.3); consequently, the integrands in eqns $(A)$ in a partially plastified element are not differentiable functions of $s$ either. This is in conflict with a basic assumption of most of common numerical integration algorithms and is the source of an additional error of numerical integration. Please note that the same problem arises with other finite element formulations. Furthermore, in the analysis of elastic-perfectly plastic frames under practically important loads the plastic hinge usually first occurs at the end points of a beam or a column element. It is important, then, that an integration point coincides with a possible plastic hinge. Lobatto's integration scheme satisfies this condition and is in this regard the preferred option. On the other hand, if material is elastic, $C_{11}$, $C_{12}$, and $C_{22}$ are constant and the preferred integration method is Gaussian, because it is more accurate than Lobatto's. A method that would correctly accounts for these problems and would work equally well in purely elastic and in plastic regime is not known. In the 
context of an elastic-plastic frame analysis, Lobatto's integration scheme was probably first used by Banovec [2]. The order of Lobatto's integration for straight beam finite element is theoretically discussed and numerically proved in [31], and is found to be equal to $M-1$. The conclusion is also valid for curved beam elements; the arguments will therefore be omitted.

\section{DETERMINATION OF INTERNAL FORCES AND DISPLACEMENTS}

An analysis of eqns (14) and (26) provides a physical meaning of functions $\lambda_{1}(s)$ and $\lambda_{2}(s)$ : these are the cross-sectional internal forces with respect to the global coordinate system $(x, z)$. Therefore, once $\varepsilon(s), \varphi(s), \lambda_{1}(s)$, and $\lambda_{2}(s)$ are obtained as solutions of eqns $(14),(26)$ and (37), the internal axial and shear forces, $\mathcal{N}(s)$ and $\mathcal{Q}(s)$, respectively, with respect to the element material coordinate system $(\xi, \eta, \zeta)$ can be evaluated by the coordinate transformation equations

$$
\begin{aligned}
& \mathcal{N}(s)=\lambda_{1}(s) \cos \varphi(s)-\lambda_{2}(s) \sin \varphi(s), \\
& \mathcal{Q}(s)=\lambda_{1}(s) \sin \varphi(s)+\lambda_{2}(s) \cos \varphi(s) .
\end{aligned}
$$

The internal bending moment is determined from an equilibrium eqn (15)

$$
\mathcal{M}(s)=-S_{3}+\int_{0}^{s}\left\{[1+\varepsilon(\xi)] \mathcal{Q}(\xi)-m_{y}(\xi)\right\} d \xi
$$

It is important to notify that the internal forces evaluated by (40)-(42) are determined from global and local equilibrium conditions and are termed equilibrium internal forces. An alternative is using eqns (10) and (11), which requires the evaluation of extensional strain $D$ and the integration of stress $\sigma$ over the cross-section. As already mentioned, these alternative internal forces are termed cross-sectional stress-resultants. Since eqn (14) is fully satisfied, cross-sectional axial force $\mathcal{N}_{c}$ equals equilibrium axial force $\mathcal{N}$, i.e., $\mathcal{N}_{c}=\mathcal{N}$. In contrast, eqn (15) is in the present approach satisfied in an integral sense only, thereby a discrepancy between cross-sectional bending moment $\mathcal{M}_{c}$ and equilibrium bending moment $\mathcal{M}$ along the axis of the beam usually emerges. For a linear elastic material, the discrepancy is often negligible. For elastic-plastic material, a small but not negligible difference between the two moments may develop when a coarse mesh is used, as shown in numerical examples.

Displacements $u(s)$ and $w(s)$ and the corresponding deformed shape of the beam are obtained by the numerical integration of kinematical eqns (3) and (4) only after the iteration 
process has been completed. The displacements are not needed in the formation of the iteration equation (37). They are computed only at those locations where desired, and with a numerical integration of a chosen accuracy.

\section{NUMERICAL EXAMPLES}

\subsection{A stability of a shallow arch: a theoretical and experimental verification}

The stability of planar circular shallow slender arches with a radius of curvature $R=254 \mathrm{~cm}$ was theoretically and experimentally studied by Dickie and Broughton [9]. The arches were made from thin perspex (polymethylmethacrylate) strip and a series of tests were conducted [9]. Three cases with different support conditions were considered (Fig. 3): case (1) both supports are hinged; case (2) one support hinged, one clamped; case (3) both supports are clamped. Two different rectangular cross-sections were used in analyses: case (a) the height of the cross-section, $h$, is $0.95 \mathrm{~cm}$; (b) the height of the cross-section is $1.27 \mathrm{~cm}$. Various types of radial loading, including a vertical point load at the center of the arch, were applied. Only the results for the center load are compared in the present paper.

The arch is modeled by the four element finite element mesh. The number of interpolation points is taken to be $M=6$. The integration over the cross-section is analytical. The integration along the element axis is performed by Lobatto's rule using five integration points.

In [9] the exact theoretical results for the critical load, considering only elastic material law, mostly overestimated the critical load obtained by their experiments. This may in part be due to an experimental error. As reported in [9], the experimental critical loads given in the paper represent the mean values obtained from two separate tests with the variation being as high as $\pm 5 \%$. Another cause of the discrepancy might be due to inelasticity of material. Therefore, our numerical analyses have been performed for elastic and elastic-perfectly plastic material. The stress-strain diagram of material and extensional yield strain $D_{Y}$ are not given in [9]. The latter is taken to be 0.004 in our calculations. The results for critical loads reported in [9] are compared to our results in Table 1. The results obtained by elastic model compare well with those reported in [9] as exact theoretical results. In contrast, results obtained by the experiments differ from elastic-model results. If elastic-perfectly plastic material model is considered, the discrepancy between the experimental and numerical results decreases. For example, in cases (2b) and (3b) differences are as high as $10 \%$, if elastic model is assumed, and only $1 \%$, if elastic-plastic model is used. The error decreases in cases (2a) and (3a), too. For two-hinged arches, cases (1a) and (1b), however, the effect of plasticity is negligible. A 
similar, yet even more pronounced overestimation of the theoretical critical load for an elastic shallow clamped arch subjected to a point load, when compared to an experimental value, was reported in [38].

\subsection{A stability of a deep circular arch}

The elastic stability of a slender clamped-hinged deep arch has been among first studied by DaDeppo and Schmidt [8] under the assumptions of axial inextensibility and shear rigidity (Fig. 4). This example is characterized by large pre-buckling asymmetrical displacements. The numerical solution given in [8] is very accurate and had served as a reference solution in many papers (e.g. [3], [11], [14], [15], [16], [18], [33], [35], [37], [43], [45]). In [8], and as explained in [7], a combination of a finite-difference method and a Runge-Kutta procedure was used, employing a very dense finite difference mesh of 200 intervals. The error of the critical load determined by their method is estimated to be roughly $0.03 \%$ [7].

In our case, the arch is modeled by the meshes of 2,4 , or 8 finite elements. The number of interpolation points, $M$, in each element is taken to be 9. The 8-point Gaussian integration is employed along the beam axis. The integration over the cross-section is analytical.

Several authors reported the finite element solution of the problem. Their results for the critical load and the results of the present study are displayed in Table 2. As it can be seen, the present method gives the most accurate results while employing the least number of degrees of freedom. The results are accurate even though the number of elements is as low as four. For four or eight element meshes, the results coincide in five significant figures. It is therefore assumed that the result for critical load, $P_{c r}=897.29 \mathrm{kN}$, is correct to five significant figures. The relative difference between this result and the one given in [8] is $0.03 \%$ which is just the error expected in $[7,8]$. The relative error of the critical load obtained in works cited in Table 2 ranges from $0.00 \%$ to $4.54 \%$. The deformed shape of the arch prior to buckling is shown in Fig. 4; observe that the associated vertical displacement of the apex is roughly $1.137 R$.

\subsection{Elastic-plastic behavior of a ring under a cyclic force}

This problem has thoroughly been examined by Coulter and Miller [6]. A ring of a square cross-section $(1.27 \mathrm{~cm} \times 1.27 \mathrm{~cm})$ shown in Fig. 5 is submitted to two opposite compression forces. The maximum force applied on the ring is $17.79 \mathrm{kN}$. The load is at first increased in ten equal load steps, and then decreased in ten equal steps to zero. Material is taken to be elastic-plastic. In [6] a three-linear stress-strain model has been applied. In the present study, the latter is substituted by an approximately equivalent bilinear model. The kinematic 
hardening is assumed. Due to the symmetry of the ring, only a quarter of the ring is modeled with $1,2,4,8$, or 16 curved finite elements. Elements with six interpolation points are used $(M=6)$.

Due to the elastic-plastic material model, Lobatto's integration formulae are taken with five integration points. Lobatto's five-point integration is used over the cross-section, too. The cross-section is modeled by one, two or four equal subsections, respectively.

The load-vertex deflection diagram obtained by one and 16-element meshes is compared to those obtained in [6] using 200 elements, and shown in Fig. 6. There is a very good agreement between the results.

The results for the vertex deflection obtained with different numbers of elements and different numbers of subsections are shown in Table 3. The final vertex deflection after the complete unloading $(P=0)$ is examined (Fig. 7). The vertex displacement obtained by using four elements and two sub-sections coincides with the displacement $2.9256 \mathrm{~cm}$ obtained by the most refined mesh (16 elements and 4 sub-sections) in four significant figures. The relative error is $0.026 \%$. The error obtained by using one element and one sub-section is only $10.6 \%$. The vertex deflection reported by Coulter and Miller [6] is $2.8321 \mathrm{~cm}$ (the relative difference is $3.2 \%$ ). However, it can be seen from Table 3 that the results do not converge monotonically to a limiting value. This is because the integrands of the cross-section integrals are not continuous functions of $\zeta$, and the integrands of the axial integrals are not differentiable functions of $s$. Consequently, the monotonic convergence of the numerical integration is not assured (see the relevant discussion in [31]). In Fig. 8 the variation of functions $1 / C_{11}$ and $C_{22}-C_{12}^{2} / C_{11}$, which occur in eqn (37) (see also Appendix), along the length of the quarter of the ring (Fig. 5) for four elements and four sub-sections finite element mesh are shown at $P=17.79 \mathrm{kN}$. Observe that the two functions which appear to be the multiplication factors in integrands of the coefficients of the system of eqn (37) (see Appendix) are not differentiable. Therefore, the coefficients of the tangential stiffness matrix of a partially plastified element can not be evaluated with monotonic convergence rate by standard integration methods.

When interpreting results of an elastic-plastic analysis of beams, it is important to notify the difference between the cross-sectional resultants $\left(\mathcal{N}_{c}\right.$ and $\left.\mathcal{M}_{c}\right)$ and the equilibrium axial force and the bending moment $(\mathcal{N}$ and $\mathcal{M})$. The cross-sectional resultants are evaluated by the integration of eqns (10) and (11), whereas the equilibrium axial force and bending moment are obtained from eqns (40) and (42). By a comparison of eqns (14) and (40), we observe that the cross-sectional resultant and equilibrium axial forces are equal: $\mathcal{N}_{c}=\mathcal{N}$. A difference 
arises in the case of the bending moment only. For the sake of error estimation, the bending moments at points $A, B$, and $C$ (see Fig. 5) at the maximum load factor are considered. The values obtained by three different finite element meshes are shown in Table 4 . The crosssectional resultant bending moment is not continuous over the boundaries of an element, whereas the equilibrium bending moment is. Therefore, there are two different values of the cross-sectional bending moment at mid-point $C$, as shown in Table 4 . The results show that the discrepancy is rather big at point $C$, yet negligible at points $A$ and $B$, even though a very coarse finite element mesh of two elements is used. For refined meshes (4 and 16 elements), the difference becomes negligible. Furthermore, it can be seen that the equilibrium bending moment but not the cross-sectional one is an excellent approximation even if a very coarse mesh is used.

The circular ring has also been modeled by 4 and 16 elements describing the whole ring. Two types of boundary conditions have been applied: (a) a clamped support at the bottom of the ring (Fig. 9a), and (b) the same as in (a) but an additional support preventing sway has been added (Fig. 9b). The results obtained by two supports - full ring model coincide precisely with the results obtained by the quarter ring model. In case (a) the results coincide only for first three load increments. Then an asymmetrical buckling takes place at the load $5.713 \mathrm{kN}$, which is roughly $32 \%$ of the maximum load of case (b). At this point a horizontal in-plane sway occurs. The same value of the critical load $(5.713 \mathrm{kN})$ has been obtained by both finite element meshes (4 and 16 elements).

Numerical experimentations prove a quadratic rate of convergence of global Newton's iteration in elastic and plastic analyses. Four or five iterations are usually required to achieve the accuracy $10^{-9}$ of nodal displacements and rotations.

\subsection{A clothoid shaped spring}

A clothoid shaped spring of length $L$ is clamped at one end and loaded by a point moment $\mathcal{M}_{B}$ at the other (Fig. 10). The spatial coordinates of its centroid axis are defined by the parametric equations

$$
x(s)=\int_{0}^{s} \cos \frac{u^{2}}{2 a^{2}} d u, \quad z(s)=\int_{0}^{s} \sin \frac{u^{2}}{2 a^{2}} d u
$$

where $a$ is chosen to be 1 . The clothoid is characterized by a linear dependence of the initial curvature on $s$. Material is taken to be bilinear elastic-plastic.

The problem is first solved analytically. It is easy to see that the axial and the shear forces are equal to zero, whereas the bending moment is constant along the spring and equal to 
applied external moment $\mathcal{M}_{B}$. Therefore, extensional strain $\varepsilon$ of the centroid axis is also equal to zero. The governing differential equation (15) takes a simple form

$$
\mathcal{M}_{c}^{\prime}\left(\Delta \varphi^{\prime}\right)=0, \quad \text { where } \quad \Delta \varphi^{\prime}=\varphi^{\prime}-\varphi_{0}^{\prime}
$$

The related boundary conditions are

$$
\begin{aligned}
& \mathcal{M}_{c}\left(\Delta \varphi^{\prime}(L)\right)=\mathcal{M}_{B}, \\
& \varphi(0)=0 .
\end{aligned}
$$

Once the constitutive relation $\sigma=\sigma(D)$ is assumed, a functional relationship between $\mathcal{M}_{c}$ and $\Delta \varphi^{\prime}$ can be derived for different cross-sections. For the case of a rectangular cross-section (width $b$ and height $h$ ), the relationship (11) can be proved to assume the form

$$
\mathcal{M}_{c}\left(\Delta \varphi^{\prime}\right)=\left\{\begin{array}{ll}
\Delta \varphi^{\prime} \frac{b h^{3}}{12} E, & \left|\Delta \varphi^{\prime}\right| \leq 2 \frac{D_{Y}}{h} \\
D_{Y}\left(E-E_{p}\right)\left(\frac{h^{2}}{4}-\frac{D_{Y}^{2}}{3\left(\Delta \varphi^{\prime}\right)^{2}}\right) b \operatorname{sgn} \Delta \varphi^{\prime}+\Delta \varphi^{\prime} \frac{b h^{3}}{12} E_{p}, & \left|\Delta \varphi^{\prime}\right|>2 \frac{D_{Y}}{h}
\end{array} .\right.
$$

By considering boundary conditions (45) and (46) and after solving of eqns (44) and (47), we obtain

$$
\Delta \varphi^{\prime}\left(\mathcal{M}_{B}\right)=\text { const }
$$

This shows that the change of rotation is a linear function of $s$ and that the deformed shape of the plastified spring retains the characteristics of the clothoid.

Different finite element meshes (one, two, and three curved elements, with $M=6$ interpolating points) have been employed and the results compared to analytical result obtained from eqn (48). The integration over the cross-section has been performed either analytically or numerically (using one or two sub-sections, Gaussian rule, and 10 integration points). The integration along the length of the element is Gaussian with 10 integration points. Moment $\mathcal{M}_{B}$ increases from 0 to the final value 2.4 in eight equal increments $\left(\Delta \mathcal{M}_{B}=0.3\right)$. The descriptive data are given in Fig. 10. All data and results are presented in consistent physical units.

The plastification occurs first at the outer fibres at $\mathcal{M}_{B}=1.225$. For $\mathcal{M}_{B}$ smaller than 1.225, the rotation as well as tip displacements obtained by FEM analysis coincide with the analytical results regardless of the finite element mesh used. Results corresponding to a very large load $\mathcal{M}_{B}=2.4$ are, however, less accurate. The rotation of the centroid axis at the 
free end of the spring is considered first. When the analytical integration over the crosssection has been used, the FEM results, regardless of the number of elements, coincide with analytical ones (see Table 5). Note that the change of the tip rotation is extremely large, $\Delta \varphi_{B}=60.87 \approx 19 \pi$ (see Figs. 11 and 13 ). The results are less accurate, though, if the tip displacements are considered (see Table 5). In the present numerical example, displacements evaluated by FEM are virtually exact, if the three-element mesh in conjunction with the analytical integration over the cross-section is used. The results in Table 5 further indicate the importance of an accurate cross-sectional integration.

In Fig. 11 the relation between the change of the tip rotation and the applied moment is shown. In elastic region, i.e., for the applied moment $\mathcal{M}_{B}$ lower than 1.225 , the relation is linear even though displacements and rotations are large (the change of rotation $\Delta \varphi_{B}$ for $\mathcal{M}_{B}=1.225$ is 9.80). After the first plastification occurs, the relation becomes nonlinear. The relation between tip displacements and the applied moment is shown in Fig. 12. There is no evident part for which the linear relation may be assumed. Yet it is interesting that both displacements approach the value of approximately -1.0 for a very large moment. The deformed shapes of the spring for some characteristic load steps are shown in Fig. 13. The deformed shape at the maximum load, $\mathcal{M}_{B}=2.4$, approaches a circle and is not shown for the sake of clarity.

\subsection{A diamond shaped straight-beam frame}

Here we want to show that a straight beam can accurately be modeled by the curved beam element, by simply taking the initial curvature to be zero. A diamond shaped frame, composed from four equal straight beams, and subjected to a pair of forces at hinged joints (Fig. 14a), has been solved analytically by means of elliptic integrals for elastic material by Jenkins et al. [17], whereas Mattiasson [22] presented their solution in tabular form, accurate to six significant figures. The problem has also been studied by Surana and Sorem [41]. A finite element model encompasses only a quarter of the structure (Fig. 14b). The elastic material model and the effect of plastification are considered here. Extensional yield strain $D_{Y}$ is taken to be either $0.10,0.05$, or 0.01 , and the plastic modulus of material, $E_{p}$, equals one tenth of the elastic modulus, $E_{p}=E / 10$. The kinematic hardening is considered during unloading.

One, two, and four elements with $M=6$ have been used. The numerical integration along the element axis is Gaussian with five integration points, if the elastic material model is considered. For elastic-plastic analyses, Lobatto's integration rule with five integration points 
is employed.

Elastic material is considered first. The results obtained by different finite element meshes for the elastic analysis are presented in Table 6 and compared to exact solutions of Mattiasson [22] for $P L^{2} / E I=10$. One load step is used and typically six iterations are needed to obtain the results accurate to six significant figures. The results for two and four element meshes coincide with the exact solution. The maximum relative error of one-element mesh is approximately $0.05 \%$ for the horizontal displacement of point $A$. The differences for others quantities are even smaller.

The effect of the spread of the plastification during loading and unloading on the tip deflection is shown in Fig. 15. Observe that the behavior of the diamond shaped structure subjected to the loading-unloading cycle is somehow similar to the behavior of the circular ring presented in Section 6.3.

In the present example, the straight beam has been modeled by the curved beam element, setting its initial curvature to zero. The computations show that this not only works theoretically correct, but that it is also a computationally efficient procedure. The advantage is that no transformations from local to global coordinate systems need to be done, thus enhancing the computational efficiency.

\section{CONCLUSIONS}

In this paper the finite element formulation of the geometrically nonlinear elastic-plastic static behavior of initially arbitrary curved planar beams has been presented, and a family of new computationally more efficient finite elements derived as known so far. The main characteristic of the formulation is that only one function, the rotation of the centroid axis, needs to be interpolated. Hence, these elements are inherently locking-free and need less degrees of freedom for a given accuracy. Exact kinematic equations for slender curved beams given by Reissner [28] have been employed, yet the effect of shear strains is neglected in the present formulation. However, the effect of shear may relatively easily be incorporated in this formulation, similarly as done in [30] for elastic material.

Newton's method has been applied for the solution of equilibrium equations and a quadratic convergence rate is proved in elastic as well in plastic analyses.

All of the numerical results presented show excellent accuracy and high computational efficiency of the derived elements even though the finite element mesh is very coarse and the initial curvature is not a constant. This enables modeling of beams of complicated initial 
curvature with only a few finite elements.

Experience with elastic-plastic beam analyses show that it is difficult to obtain strictly monotonicaly convergent results. The main problem lies in the numerical integration required in the tangential stiffness matrix evaluation, which in plastic region has non-differentiable integrands. When the integrand is not differentiable, standard numerical integration schemes are not capable of giving monotonicaly convergent results for reasonably low numbers of integration points. The only means of solution to this problem is to identify the points of integrand's slope discontinuity and to integrate by subregions. However, such a solution scheme would not be easily applicable and is computationally very demanding.

An advantage of our finite elements is also the way the internal forces are determined. The evaluation of the internal forces does not involve the differentiation of basic variables. Therefore, the internal forces are obtained with the same degree of accuracy as basic variables, i.e., displacements and rotations. This is shown in a numerical example. Moreover, it is shown that an internal force evaluated alternatively by a differentiation of basic variables may differ considerably in plastic regions compared to the more accurate internal force evaluated from equilibrium equations. Furthermore, internal forces evaluated from equilibrium equations satisfy the continuity condition over the beam element border where required, which is not the case if internal forces are evaluated otherwise.

The essential unknown variables of the finite element correspond to the fixed-in-space coordinate system of a structure. The coordinate transformation from the local to the global coordinate system is therefore not needed. This feature makes the formulation computationally still more attractive and efficient, especially when compared to the corotational formulations, and greatly facilitate the implementation of the present finite element formulation in the sensitivity analysis [18].

Acknowledgements - The work has been financially supported by the Ministry of Science and Technology of the Republic of Slovenia under contract J2-6195-792. The support is gratefully acknowledged. The authors would like to thank I. Planinc, M.Sc., University of Ljubljana, for helpful discussions and useful suggestions.

\section{REFERENCES}


[1] C. R. Babu and G. Prathap, A linear thich curved beam element. Int. J. Numer. Meth. Engng. 23, 1313-1328 (1986).

[2] J. Banovec, An efficient finite element method for elastic-plastic analysis of plane frames. Nonlinear Finite Element Analysis in Structural Mechanics (T. Wunderlich, E. Stein and K.-J. Bathe, eds.), Springer, 385-402 (1981).

[3] M. Borri and C. Bottasso, An intrinsic beam model based on a helicoidal approximation - Part II: Linearization and finite element implementation. Int. J. Numer. Meth. Engng. 37, 2291-2309 (1994).

[4] C. J. Burgoyne and M. A. Crisfield, Numerical integration strategy for plates and shells. Int. J. Numer. Meth. Engng. 18, 105-121 (1990).

[5] J. K. Choi and J. K. Lim, General curved beam elements based on the assumed strain fields. Comput. Struct. 55, 379-386 (1995).

[6] B. A. Coulter and R. E. Miller, Loading, unloading and reloading of a generalized plane plastica. Int. J. Numer. Meth. Engng. 28, 1645-1660 (1989).

[7] D. A. DaDeppo and R. Schmidt, Large deflections and stability of hingless circular arches under interacting loads. ASME J. Appl. Mechanics 96, 989-994 (1974).

[8] D. A. DaDeppo and R. Schmidt, Instability of clamped-hinged circular arches subjected to a point load. ASME J. Appl. Mechanics 97, 894-896 (1975).

[9] J. F. Dickie and P. Broughton, Stability criteria for shallow arches. ASCE J. Eng. Mech. Div. 97, 951-965 (1971).

[10] E. N. Dvorkin, E. Oñate and J. Oliver, On a non-linear formulation for curved Timoshenko beam elements considering large displacement/rotation increments. Int. J. Numer. Meth. Engng. 26, 1597-1613 (1988).

[11] C. G. Franchi and F. Montelaghi, A weak-weak formulation for large displacements beam statics: a finite volumes approximation. Int. J. Numer. Meth. Engng. 39, 585-604 (1996).

[12] C. Gontier and C. Vollmer, A large displacement analysis of a beam using a CAD geometric definition. Comput. Struct. 57, 981-989 (1995). 
[13] Y. Goto, T. Yamashita and S. Matsuura, Elliptic integral solutions for extensional elastica with constant initial curvature. JSCE Structural Eng./Earthquake Eng. 4, 299-309 (1987).

[14] K. M. Hsiao and F. Y. Hou, Nonlinear finite element analysis of elastic frames. Comput. Struct. 26, 693-701 (1987).

[15] A. Ibrahimbegović, On finite element implementation of geometrically nonlinear Reissner's beam theory: three-dimensional curved beam elements. Comput. Methods Appl. Mech. Engng. 122, 11-26 (1995).

[16] A. Ibrahimbegović and F. Frey, Finite element analysis of linear and non-linear planar deformations of elastic initially curved beams. Int. J. Numer. Meth. Engng. 36, 32393258 (1993).

[17] J. A. Jenkins, T. B. Seitz and J. S. Przemieniecki, Large deflections of diamond-shaped frames. Int. J. Solids Struct. 2, 591-603 (1966).

[18] M. Kegl, B. J. Butinar and M. M. Oblak, Shape optimal design of elastic planar frames with non-linear response. Int. J. Numer. Meth. Engng. 38, 3227-3242 (1995).

[19] B. L. Koziey and F. A. Mirza, Consistent curved beam element. Comput. Struct. 51, 643-654 (1994).

[20] P.-G. Lee and H.-C. Sin, Locking-free curved beam element based on curvature. Int. J. Numer. Meth. Engng. 37, 989-1007 (1994).

[21] S.-S. Lee, J. S. Koo and J. M. Choi, Development of a new curved beam element with shear effect. Eng. Comput. 13, 9-25 (1996).

[22] K. Mattiasson, Numerical results from large deflection beam and frame problems analysed by means of elliptic integrals. Int. J. Numer. Meth. Engng. 16, 145-153 (1981).

[23] A. K. Noor and J. M. Peters, Mixed models and reduced/selective integration displacement models for nonlinear analysis of curved beams. Int. J. Numer. Meth. Engng. 17, 615-631 (1981).

[24] A. K. Noor, W. H. Greene and S. J. Hartley, Nonlinear finite element analysis of curved beams. Comp. Meth. Appl. Mech. Engng. 12, 289-307 (1977). 
[25] R. Y. S. Pak and E. J. Stauffer, Nonlinear finite deformation analysis of beams and columns. ASCE J. Eng. Mech. 120, 2136-2153 (1994).

[26] S. J. Pantazopoulou, Low-order interpolation functions for curved beams. ASCE J. Eng. Mech. 118, 329-350 (1992).

[27] B. D. Reddy and M. B. Volpi, Mixed finite element methods for the circular arch problem. Comp. Meth. Appl. Mech. Eng. 97, 125-145 (1992).

[28] E. Reissner, On one-dimensional finite-strain beam theory: the plane problem. J. Appl. Math. Phys. (ZAMP) 23, 795-804 (1972).

[29] M. Saje, A variational principle for finite planar deformation of straight slender elastic beams. Int. J. Solids Struct. 26, 887-900 (1990).

[30] M. Saje, Finite element formulation of finite planar deformation of curved elastic beams. Comput. Struct. 39, 327-337 (1991).

[31] M. Saje, I. Planinc, G. Turk and B. Vratanar, A kinematically exact finite element formulation of planar elastic-plastic frames. Comp. Meth. Appl. Mech. Engng. (to be published).

[32] A. F. Saleeb and T. Y. Chang, On the hybrid-mixed formulation of $C^{0}$ curved beam elements. Comp. Meth. Appl. Mech. Engng. 60, 95-121 (1987).

[33] J. S. Sandhu, K. A. Stevens and G. A. O. Davies, A 3-D, co-rotational, curved and twisted beam element. Comput. Struct. 35, 69-79 (1990).

[34] A. Shinohara and M. Hara, Large deflection of a circular C-shaped spring. Int. J. Mech. Sci. 21, 179-186 (1979).

[35] J. C. Simo, K. D. Hjelmstad and R. L. Taylor, Numerical formulations of elastoviscoplastic response of beams accounting for the effect of shear. Comput. Meth. Appl. Engng. 42, 301-330 (1984).

[36] J. C. Simo, A finite strain beam formulation. The three-dimensional dynamic problem, Part I. Comput. Meth. Appl. Engng. 49, 55-70 (1985).

[37] J. C. Simo, P. Wriggers, K. H. Schweizerhof and R. L. Taylor, Finite deformation postbuckling analysis involving inelasticity and contact constraints. Int. J. Numer. Meth. Engng. 23, 779-800 (1981). 
[38] H. Stolarski and T. Belytschko, Membrane locking and reduced integration for curved elements. ASME J. Appl. Mechanics 49, 172-176 (1982).

[39] H. Stolarski and T. Belytschko, Shear and membrane locking in curved $C^{0}$ elements. Comp. Meth. Appl. Mech. Engng. 41, 279-296 (1983).

[40] K. S. Surana, Geometrically non-linear formulation for two dimensional curved beam elements. Comput. Struct. 17, 105-114 (1983).

[41] K. S. Surana and R. M. Sorem, Geometrically non-linear formulation for three dimensional curved beam elements with large rotations. Int. J. Numer. Meth. Engng. 28, 43-73 (1989).

[42] A. Tessler and L. Spiridigliozzi, Curved beam elements with penalty relaxation. Int. J. Numer. Meth. Engng. 23, 2245-2262 (1986).

[43] W. Wagner, A path-following algorithm with quadratic predictor. Comput. Struct. 39, 339-348 (1991).

[44] K. Washizu, Variational Methods in Elasticity and Plasticity. Pergamon Press, Oxford (1981).

[45] R. D. Wood and O. C. Zienkiewicz, Geometrically nonlinear finite element analysis of beams, frames, arches and axisymmetric shells. Comput. Struct. 7, 725-735 (1977).

[46] Y. -B. Yang, S. -R. Kuo and Y. -D. Cherng, Curved beam elements for nonlinear analysis. ASCE J. Eng. Mech. 115, 840-855 (1989).

[47] Z. Zhang, A note on the hybrid-mixed $C^{0}$ curved beam elements. Comp. Meth. Appl. Mech. Engng. 95, 243-252 (1992). 
Coefficients of the system of eqn (37)

Using eqns (30)-(36) and expressions derived in [31], and introducing notations $g_{m}, h_{m}$, and $\bar{C}_{22}$

$$
\begin{aligned}
& g_{m}=\frac{1}{C_{11}}\left(I_{m}^{\prime} C_{12}+I_{m} \mathcal{Q}\right), \\
& h_{m}=(1+\varepsilon) I_{m}, \\
& \bar{C}_{22}=C_{22}-\frac{C_{12}^{2}}{C_{11}},
\end{aligned}
$$

non-zero coefficients of the system of eqns (37) take the forms:

$$
\left.\begin{array}{l}
\frac{\partial f_{1}}{\partial \lambda_{1}^{0}}=-\int_{0}^{L} \frac{\cos ^{2} \varphi}{C_{11}} d s \\
\frac{\partial f_{1}}{\partial \lambda_{2}^{0}}=\frac{\partial f_{2}}{\partial \lambda_{1}^{0}}=\int_{0}^{L} \frac{\sin \varphi \cos \varphi}{C_{11}} d s \\
\frac{\partial f_{1}}{\partial \varphi_{m}}=\frac{\partial f_{2+m}}{\partial \lambda_{1}^{0}}=\int_{0}^{L}\left(g_{m} \cos \varphi+h_{m} \sin \varphi\right) d s \\
\frac{\partial f_{1}}{\partial U_{1}}=\frac{\partial f_{2}}{\partial U_{2}}=\frac{\partial f_{2+M+1}}{\partial \lambda_{1}^{0}}=\frac{\partial f_{2+M+2}}{\partial \lambda_{2}^{0}}=-1 \\
\frac{\partial f_{1}}{\partial U_{4}}=\frac{\partial f_{2}}{\partial U_{5}}=\frac{\partial f_{2+M+3}}{\partial \lambda_{1}^{0}}=\frac{\partial f_{2+M+4}}{\partial \lambda_{2}^{0}}=1 \\
\frac{\partial f_{2}}{\partial \lambda_{2}^{0}}=-\int_{0}^{L} \frac{\sin ^{2} \varphi}{C_{11}} d s \\
\frac{\partial f_{2}}{\partial \varphi_{m}}=\frac{\partial f_{2+m}}{\partial \lambda_{2}^{0}}=\int_{0}^{L}\left(-g_{m} \sin \varphi+h_{m} \cos \varphi\right) d s \\
\frac{\partial f_{2+i}}{\partial \varphi_{m}}=\int_{0}^{L}\left\{I_{i}^{\prime} I_{m}^{\prime} \bar{C}_{22}-\frac{C_{12}}{C_{11}}\left(I_{m} I_{i}^{\prime}+I_{m}^{\prime} I_{i}\right) \mathcal{Q}+\left[(1+\varepsilon) \mathcal{N}-\frac{\mathcal{Q}^{2}}{C_{11}}\right] I_{m} I_{i}\right\} d s
\end{array}\right\}
$$

Coefficients $C_{11}, C_{12}$, and $C_{22}$ denote tangent stiffnesses of the cross-section, $\mathcal{A}(s)$, and are obtained by integrations over the cross-section:

$$
\begin{aligned}
C_{11}(s) & =\int_{\mathcal{A}(s)} E_{t} d A \\
C_{12}(s) & =\int_{\mathcal{A}(s)} \zeta E_{t} d A \\
C_{22}(s) & =\int_{\mathcal{A}(s)} \zeta^{2} E_{t} d A
\end{aligned}
$$

$E_{t}$ denotes the uniaxial tangent modulus of material: $E_{t}=\partial \sigma / \partial D$. In an elastic region it corresponds to modulus of elasticity $\left(E_{t}=E\right)$, whereas in a plastic region it is equal to plastic hardening modulus $E_{t}=E_{p} . \mathcal{N}$ and $\mathcal{Q}$ are given by eqns (40) and (41).

Please observe the simplicity of mathematical form of expressions in these equations. 
Table 1: The critical load for a shallow arch (in N)

\begin{tabular}{lrrrrrr}
\hline \multirow{2}{*}{ Method } & \multicolumn{6}{c}{ Case } \\
\cline { 2 - 7 } & $(1 \mathrm{a})$ & $(1 \mathrm{~b})$ & $(2 \mathrm{a})$ & $(2 \mathrm{~b})$ & $(3 \mathrm{a})$ & $(3 \mathrm{~b})$ \\
\hline Exact solution (elastic) [9] & 36.5 & 85.5 & 39.6 & 93.5 & 45.4 & 106.4 \\
Experiment [9] & 42.8 & 87.7 & 38.3 & 85.5 & 43.2 & 96.2 \\
present (elastic) & 36.5 & 86.0 & 39.6 & 94.0 & 45.9 & 106.4 \\
present (elastic-plastic) & 36.5 & 85.5 & 38.7 & 85.5 & 44.5 & 96.9 \\
\hline
\end{tabular}


Table 2: The comparison between FEM results

\begin{tabular}{lccc}
\hline Method & No. of elements & Critical load & $\mid$ Error \\
& & {$[\mathrm{kN}]$} & {$[\%]$} \\
\hline Borri, Bottasso [3] & 10 & 907 & 1.08 \\
DaDeppo, Schmidt [8] & $200 \dagger$ & 897 & 0.03 \\
Franchi, Montelaghi [11] & 40 & 898.4 & 0.12 \\
Hsiao, Hou [14] & 30 & 938 & 4.54 \\
Ibrahimbegović [15] & 20 & 897.3 & 0.00 \\
Ibrahimbegović, Frey [16] & 20 & 897.5 & 0.02 \\
Kegl et al. [18] & 4 & 897.39 & 0.01 \\
Sandhu et al. [33] & 6 & 897.48 & 0.05 \\
Simo et al. [35] & 20 & 906 & 0.97 \\
Simo et al. [37] & 20 & 907 & 1.08 \\
Wagner [43] & 20 & 927 & 3.31 \\
Wood, Zienkiewicz [45] & 16 & 924 & 2.98 \\
\hline present $(M=9)$ & 2 & 904.53 & 0.81 \\
& 4 & 897.29 & 0.00 \\
& 8 & 897.29 & 0.00 \\
\hline
\end{tabular}

$\dagger 200$ refers to the number of finite difference intervals 
Table 3. Vertex deflection after the complete unloading (in $\mathrm{cm}$ )

\begin{tabular}{cccc}
\hline \multirow{2}{*}{ No. of elements } & \multicolumn{3}{c}{ No. of sub-sections } \\
\cline { 2 - 4 } & 1 & 2 & 4 \\
\hline 1 & 2.6462 & 2.6142 & 2.6180 \\
2 & 2.8377 & 2.9088 & 2.9088 \\
4 & 2.8616 & 2.9263 & 2.9268 \\
8 & 2.8529 & 2.9238 & 2.9248 \\
16 & 2.8562 & 2.9238 & 2.9256 \\
\hline
\end{tabular}


Table 4. Equilibrium $(\mathcal{M})$ and cross-sectional $\left(\mathcal{M}_{c}\right)$ bending moments (in $\mathrm{kNm}$ ) at $P=17.79 \mathrm{kN}$

\begin{tabular}{cccccccc}
\hline \multirow{2}{*}{$\begin{array}{c}\text { No. of elem. } \\
(M=6)\end{array}$} & $\mathcal{M}$ & $\mathcal{M}_{c}$ & $\mathcal{M}$ & $\mathcal{M}_{c}$ & $\mathcal{M}$ & $\mathcal{M}_{c}^{1}$ & $\mathcal{M}_{c}^{2}$ \\
\cline { 2 - 8 } & -1.378 & -1.381 & 1.610 & 1.616 & 0.0210 & 0.0075 & 0.0208 \\
2 & -1.376 & -1.375 & 1.610 & 1.611 & 0.0224 & 0.0220 & 0.0221 \\
4 & -1.377 & -1.377 & 1.610 & 1.610 & 0.0221 & 0.0221 & 0.0221 \\
\hline
\end{tabular}


Table 5. Tip rotation and displacements at $\mathcal{M}_{B}=2.4$

\begin{tabular}{|c|c|c|c|c|c|c|c|}
\hline $\begin{array}{l}\text { No. of } \\
\text { elements }\end{array}$ & $\begin{array}{l}\text { Int. over the } \\
\text { cross-section }\end{array}$ & $\Delta \varphi_{B}$ & $\begin{array}{c}\text { |error| } \\
{[\%]}\end{array}$ & $\begin{array}{l}u_{B} \\
{[\mathrm{~m}]}\end{array}$ & $\begin{array}{c}\text { |error| } \\
{[\%]}\end{array}$ & $\begin{array}{l}w_{B} \\
{[\mathrm{~m}]}\end{array}$ & $\begin{array}{c}\text { |error| } \\
{[\%]}\end{array}$ \\
\hline \multirow[t]{3}{*}{1} & analytical & 60.86994 & 0.00 & -1.052526 & 3.30 & -1.330615 & 26.71 \\
\hline & 1 sub-sect. & 63.62447 & 4.53 & -2.363520 & 117.15 & -0.132370 & 87.40 \\
\hline & 2 sub-sect. & 61.02694 & 0.26 & -1.166368 & 7.16 & -1.378778 & 31.29 \\
\hline \multirow[t]{3}{*}{2} & analytical & 60.86994 & 0.00 & -1.076654 & 1.08 & -1.044904 & 0.50 \\
\hline & 1 sub-sect. & 63.62447 & 4.53 & -1.177811 & 8.21 & -0.921814 & 12.22 \\
\hline & 2 sub-sect. & 61.02694 & 0.26 & -1.088770 & 0.03 & -1.050793 & 0.06 \\
\hline \multirow[t]{3}{*}{3} & analytical & 60.86994 & 0.00 & -1.088411 & 0.00 & -1.050181 & 0.00 \\
\hline & 1 sub-sect. & 63.62447 & 4.53 & -1.200054 & 10.26 & -0.919354 & 12.46 \\
\hline & 2 sub-sect. & 61.02694 & 0.26 & -1.101100 & 1.17 & -1.055811 & 0.54 \\
\hline \multicolumn{2}{|c|}{ exact [eqn (48)] } & 60.86994 & & -1.088418 & & -1.050164 & \\
\hline
\end{tabular}


Table 6. Displacements and internal forces at $P L^{2} / E I=$ 10. Elastic material

\begin{tabular}{ccccr}
\hline No. of elem. & $\frac{u_{A}}{L}$ & $\frac{w_{B}}{L}$ & $\varphi_{B}$ & $\frac{\mathcal{M}_{A} L}{E I}$ \\
\hline 1 & -0.12730 & 1.30589 & 1.34277 & -5.79810 \\
2 & -0.12724 & 1.30577 & 1.34277 & -5.79867 \\
4 & -0.12724 & 1.30578 & 1.34277 & -5.79866 \\
\hline exact $[22]$ & -0.12724 & 1.30578 & 1.34277 & -5.79867 \\
\hline
\end{tabular}


Fig. 1. A beam element. Global and material coordinate systems.

Fig. 2. Interpolation points of a finite beam element.

Fig. 3. A shalow circular arch.

Fig. 4. A deep circular arch.

Fig. 5. A circular ring subjected to a cyclic force $P$.

Fig. 6. A circular ring. Load--deflection relationship.

Fig. 7. Deformed shapes of a circular ring for $P=1.779 f \mathrm{kN}(f=0, \pm 1, \pm 2, \ldots, \pm 10)$.

Fig. 8. Variation of $1 / C_{11}$ and $C_{22}-C_{12}^{2} / C_{11}$ along the quarter of the ring at $P=17.79 \mathrm{kN}$.

Fig. 9. Finite element model of a complete circular ring.

Fig. 10. A clothoid shaped spring.

Fig. 11. A clothoid shaped spring. Moment - tip rotation curve.

Fig. 12. A clothoid shaped spring. Moment - tip displacements curve.

Fig. 13. Deformed shapes of a clothoid shaped spring.

Fig. 14. A diamond shaped frame and a computational model.

Fig. 15. A diamond shaped frame. Force - tip deflection curve. 


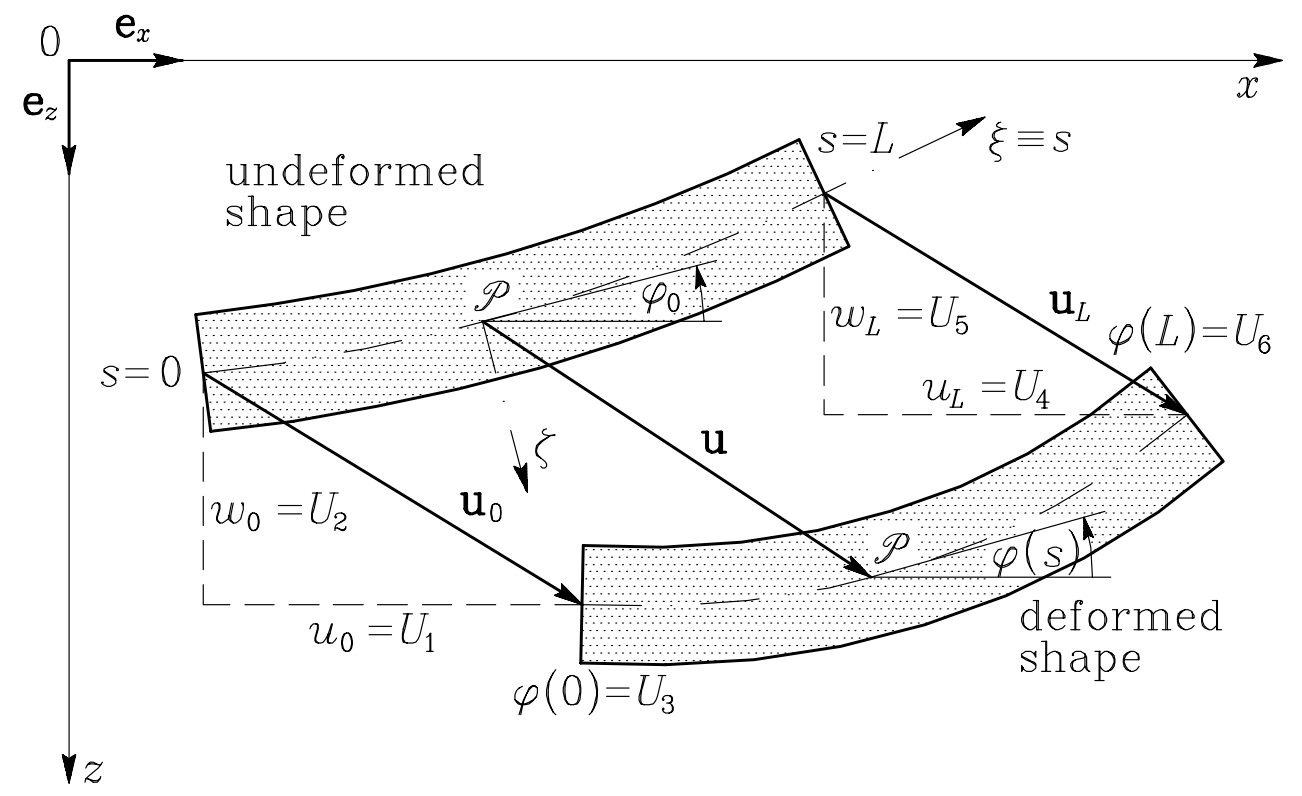

Saje, Turk, Kalagasidu, Vratanar, Figure 1. 


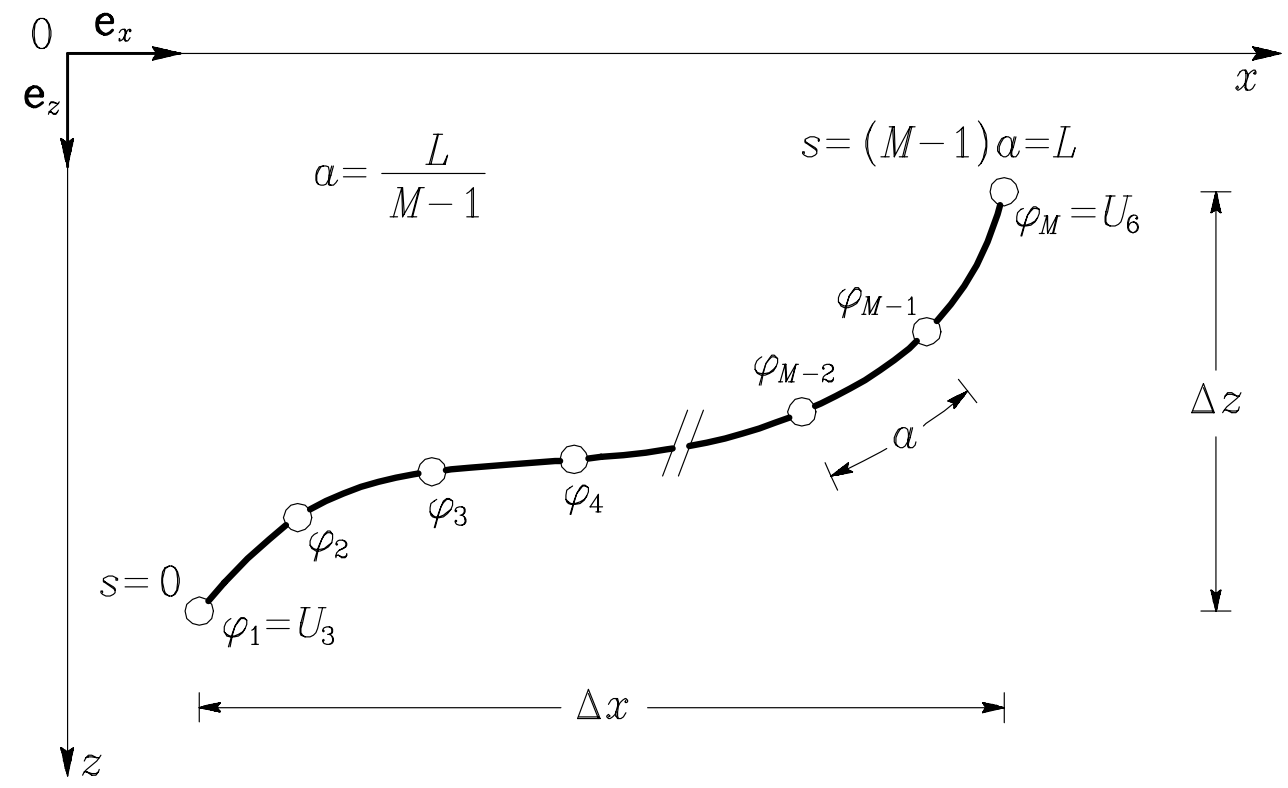

Saje, Turk, Kalagasidu, Vratanar, Figure 2. 


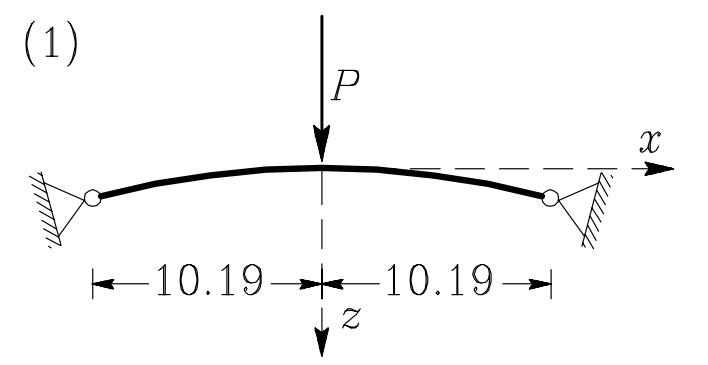

$$
\begin{aligned}
R & =254 \mathrm{~cm} \\
E & =2.9 \mathrm{GPa} \\
D_{Y} & =0.004 \quad \text { (assumed) } \\
E_{p} & =E / 10 \quad \text { (assumed) } \\
h & =0.95 \mathrm{~cm}(\mathrm{a}) \\
h & =1.27 \mathrm{~cm}(\mathrm{~b}) \\
b & =2.54 \mathrm{~cm}
\end{aligned}
$$

(2)

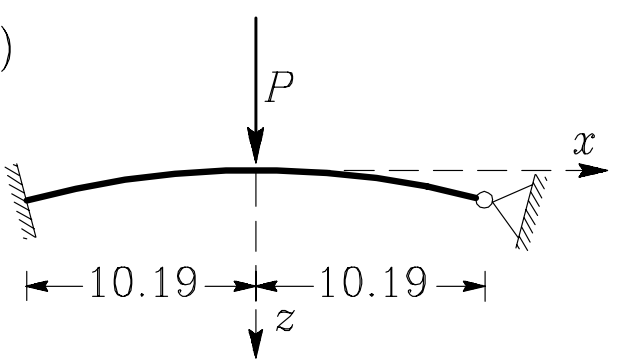

(3)

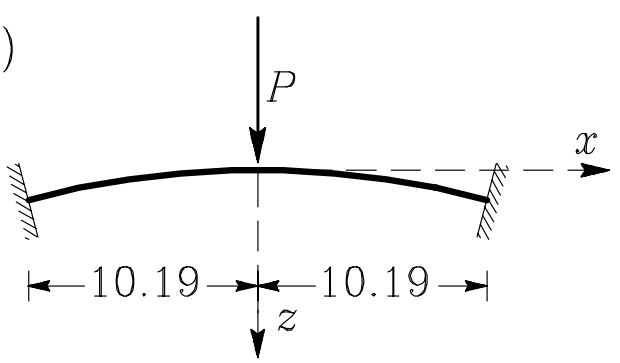

Saje, Turk, Kalagasidu, Vratanar, Figure 3. 


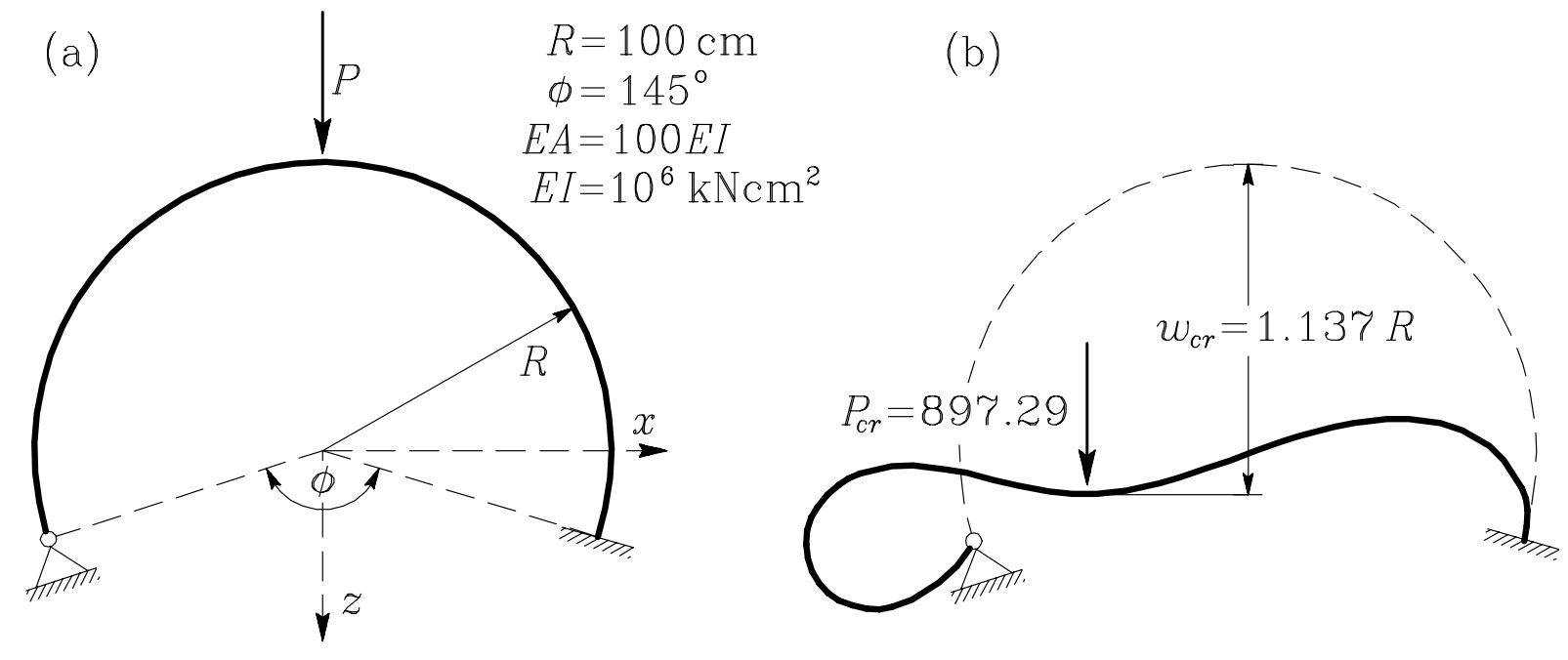

Saje, Turk, Kalagasidu, Vratanar, Figure 4. 


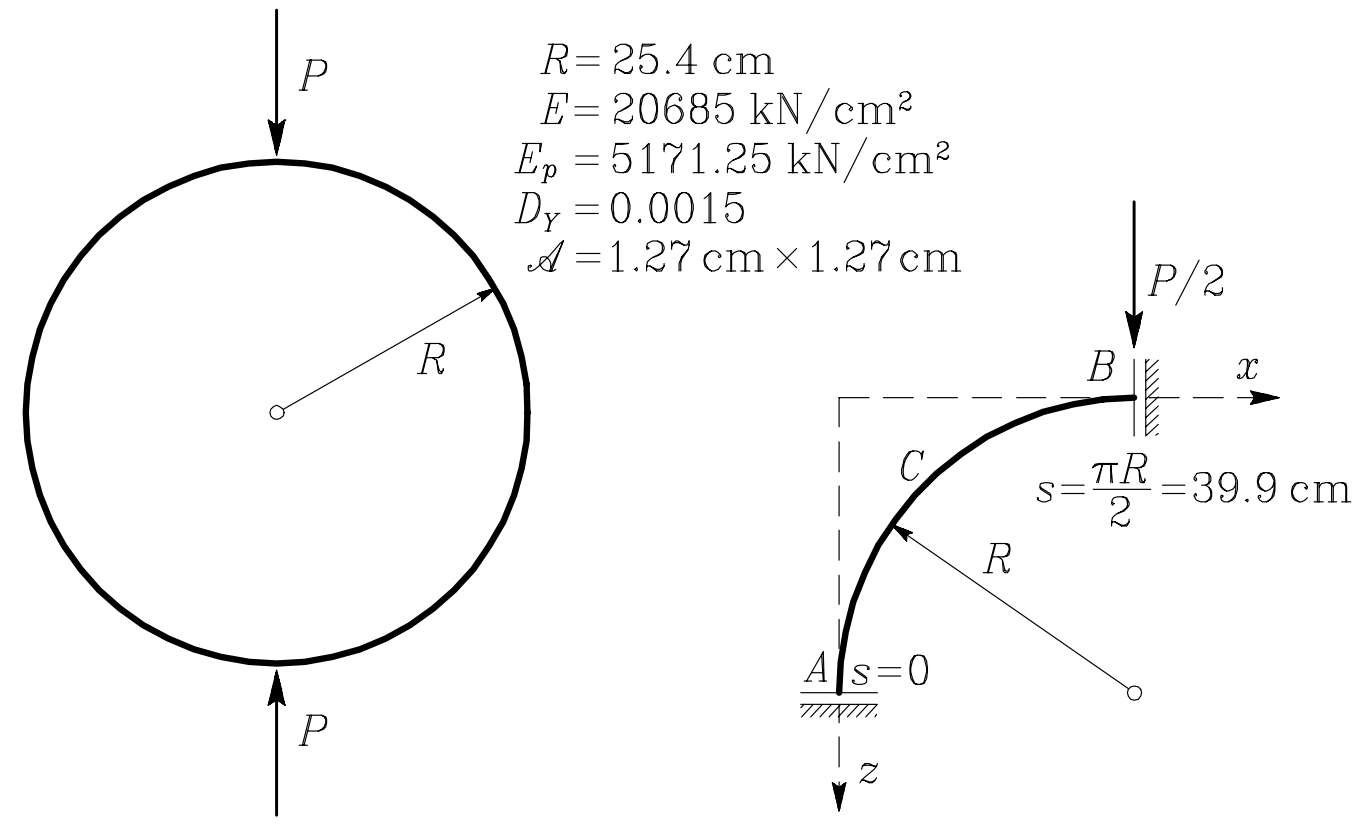

Saje, Turk, Kalagasidu, Vratanar, Figure 5. 


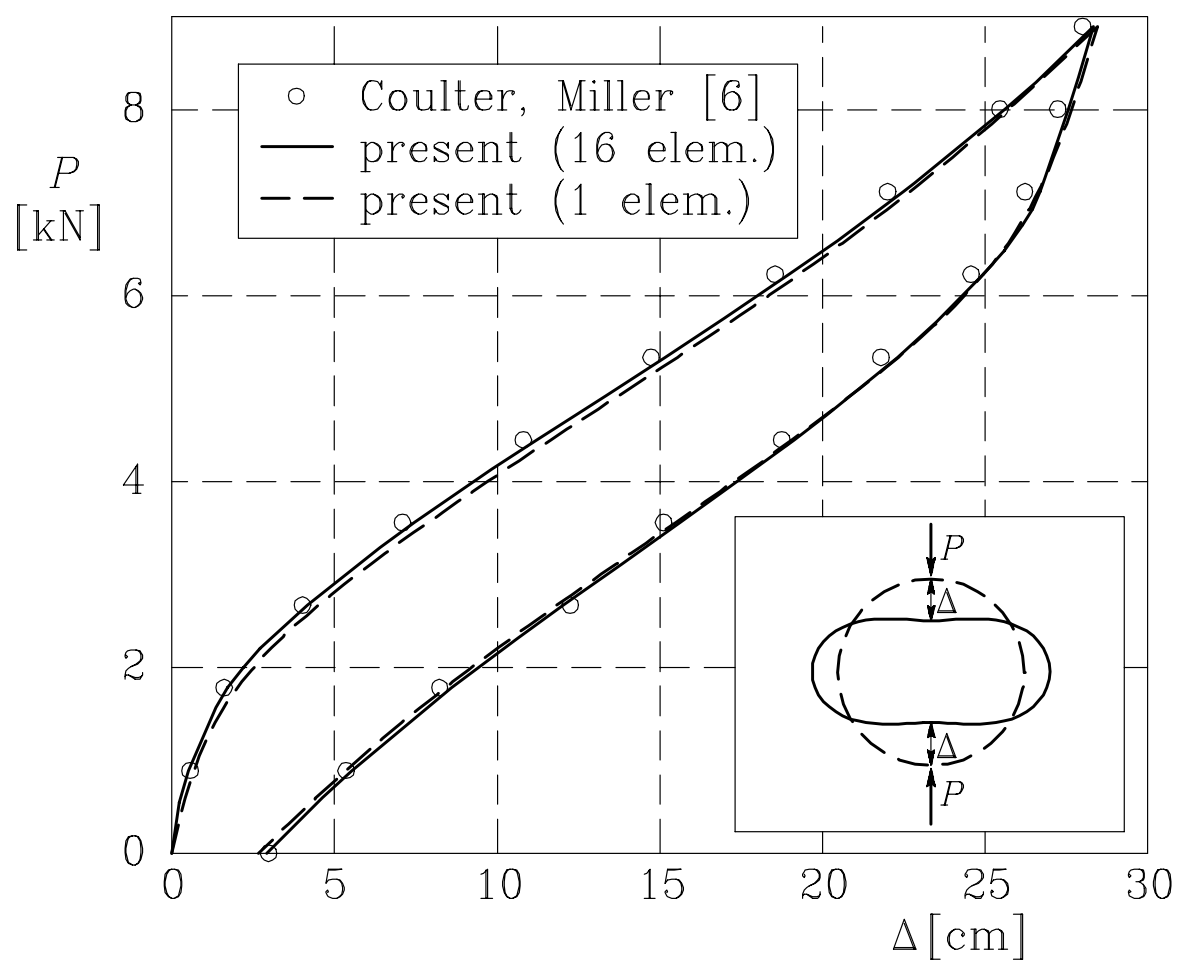

Saje, Turk, Kalagasidu, Vratanar, Figure 6. 


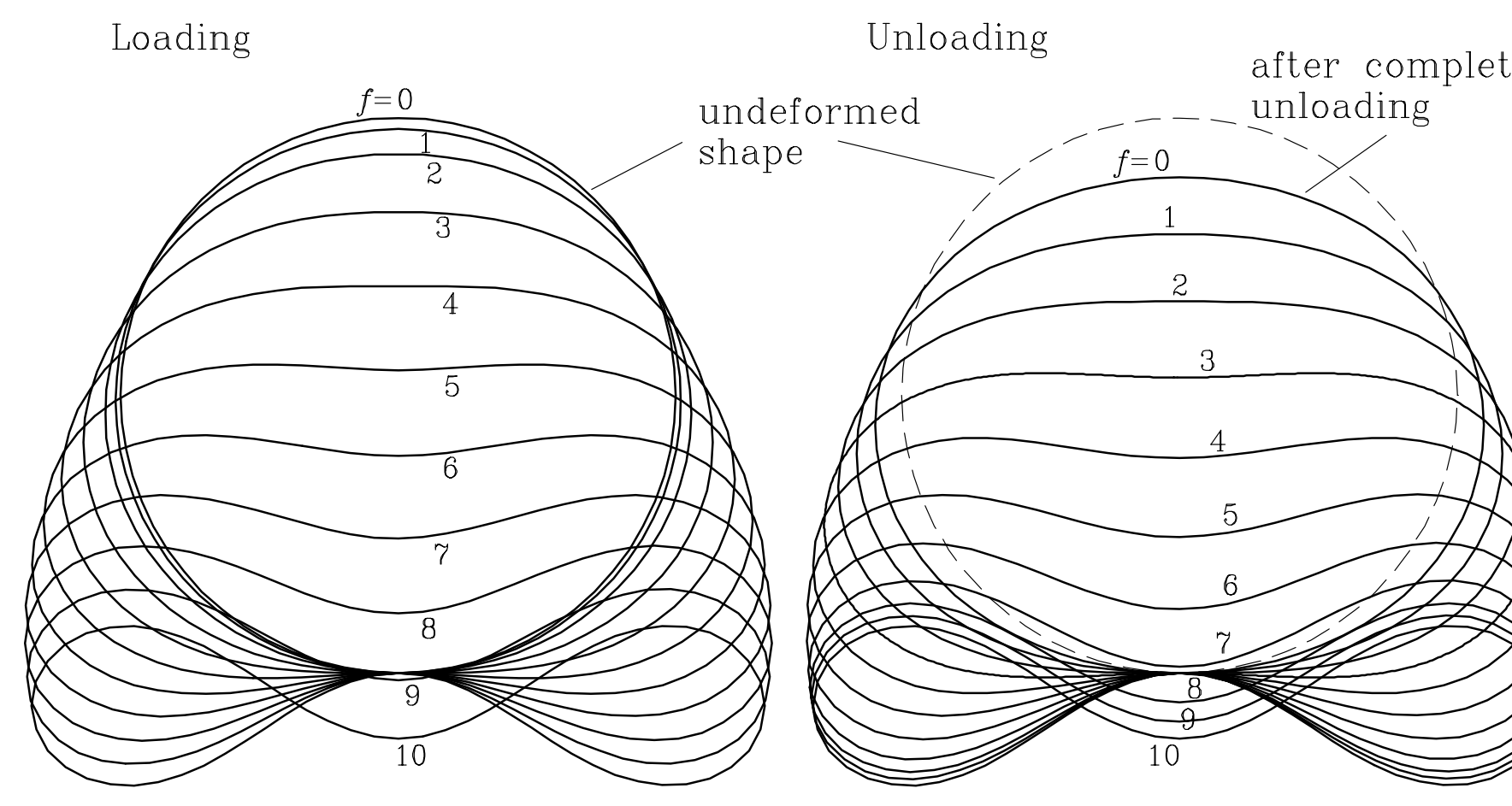

Saje, Turk, Kalagasidu, Vratanar, Figure 7. 

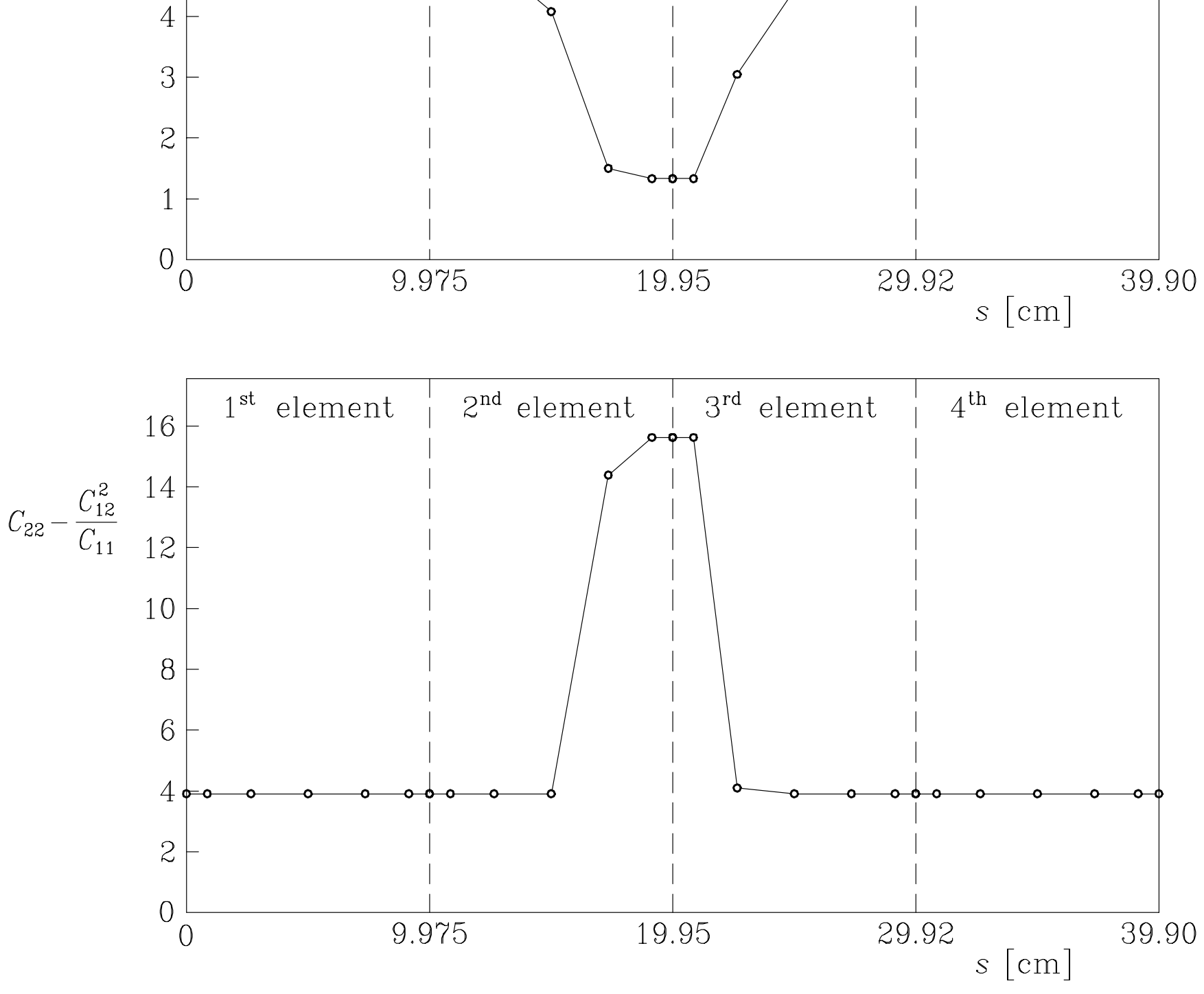

Saje, Turk, Kalagasidu, Vratanar, Figure 8. 


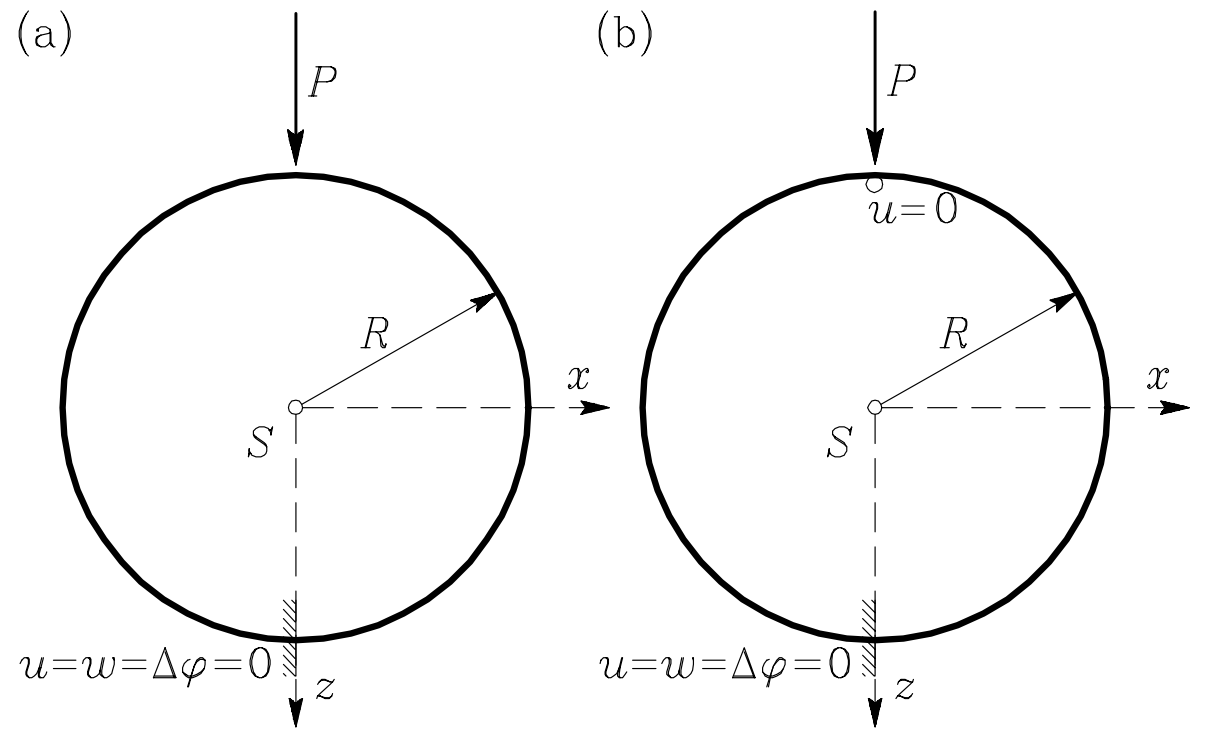

Saje, Turk, Kalagasidu, Vratanar, Figure 9. 


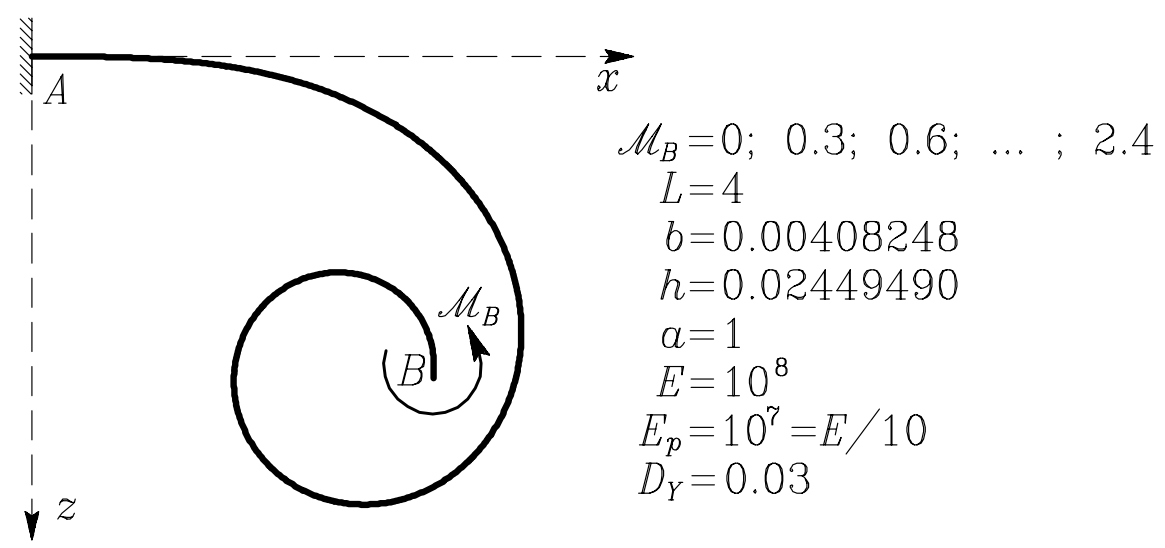

Saje, Turk, Kalagasidu, Vratanar, Figure 10. 


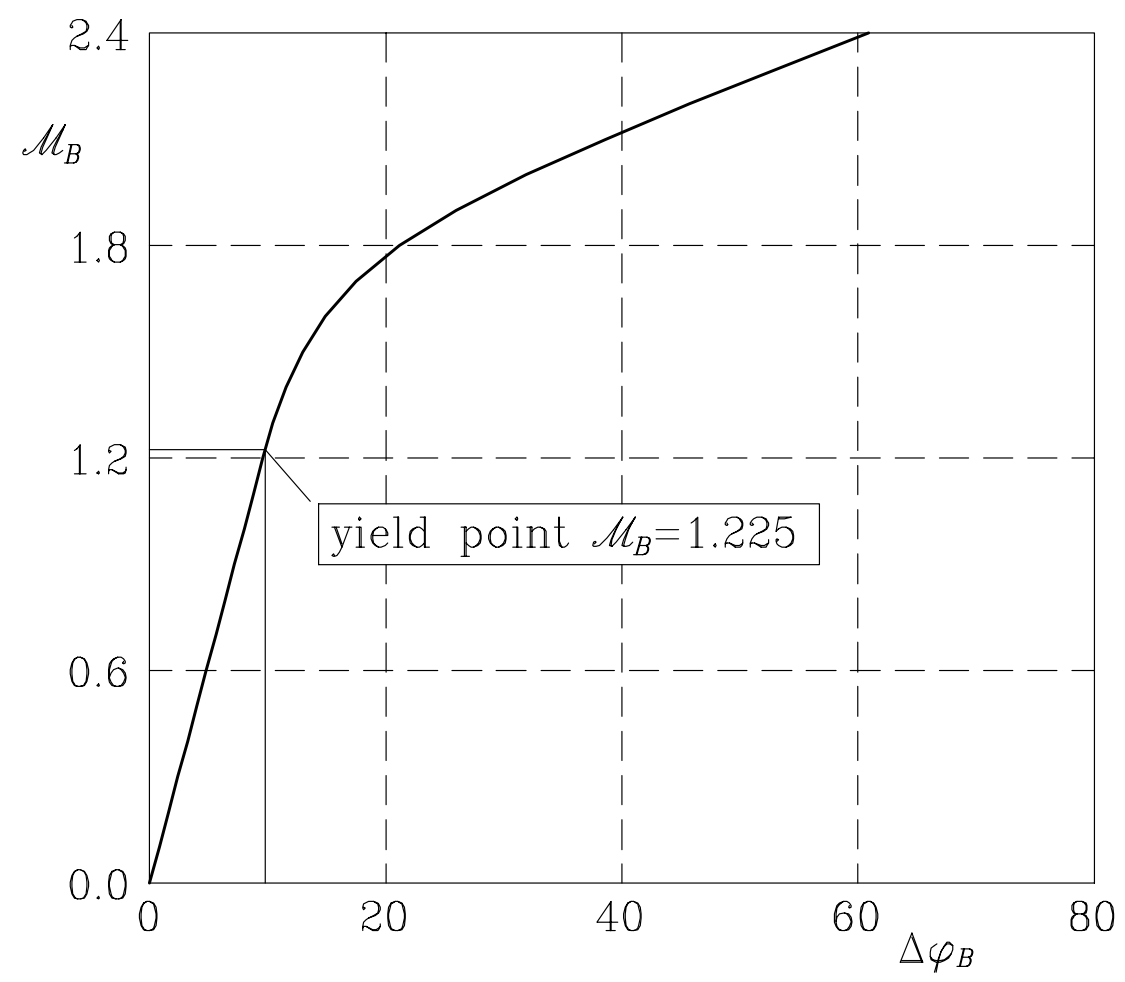

Saje, Turk, Kalagasidu, Vratanar, Figure 11. 


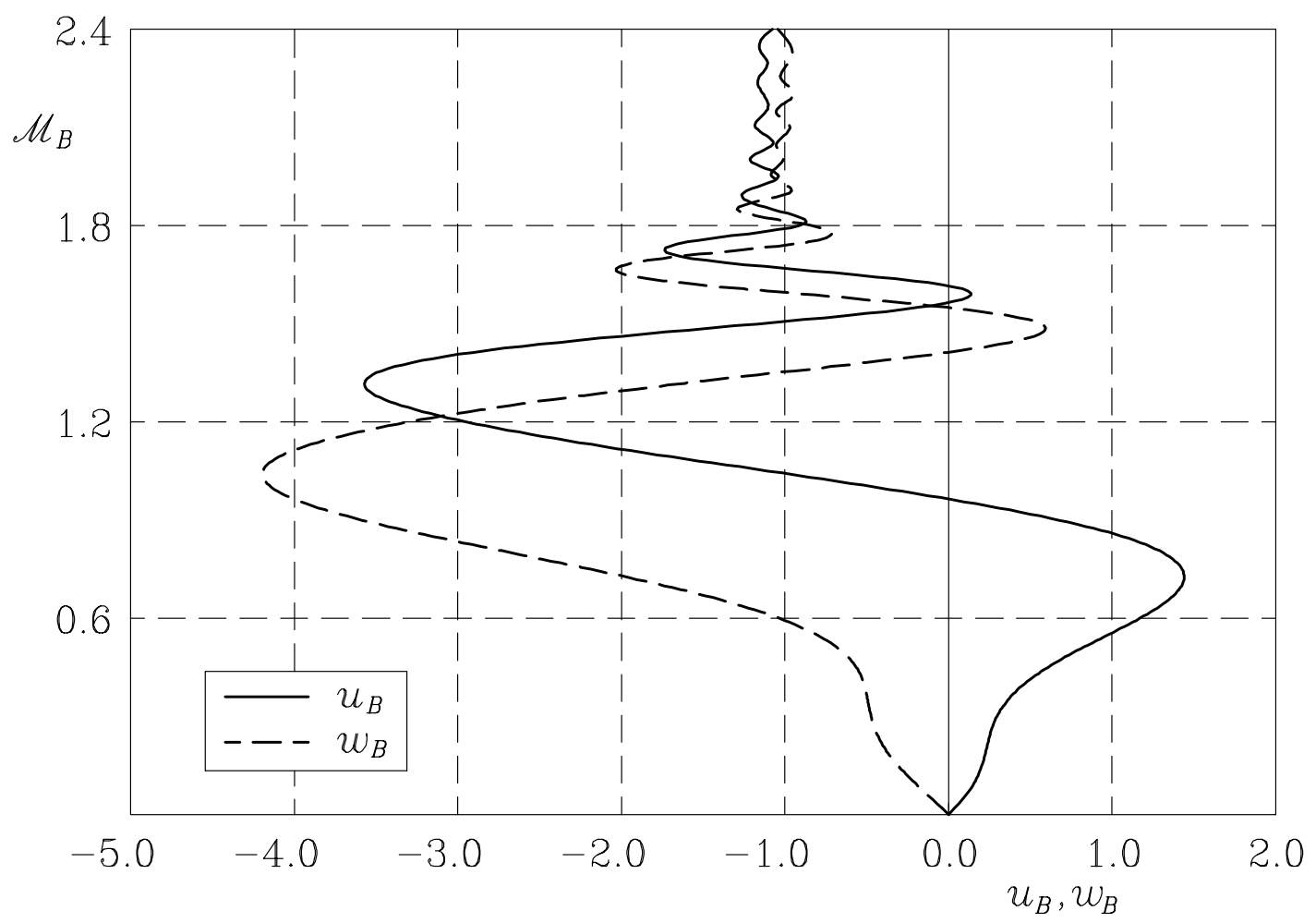

Saje, Turk, Kalagasidu, Vratanar, Figure 12. 


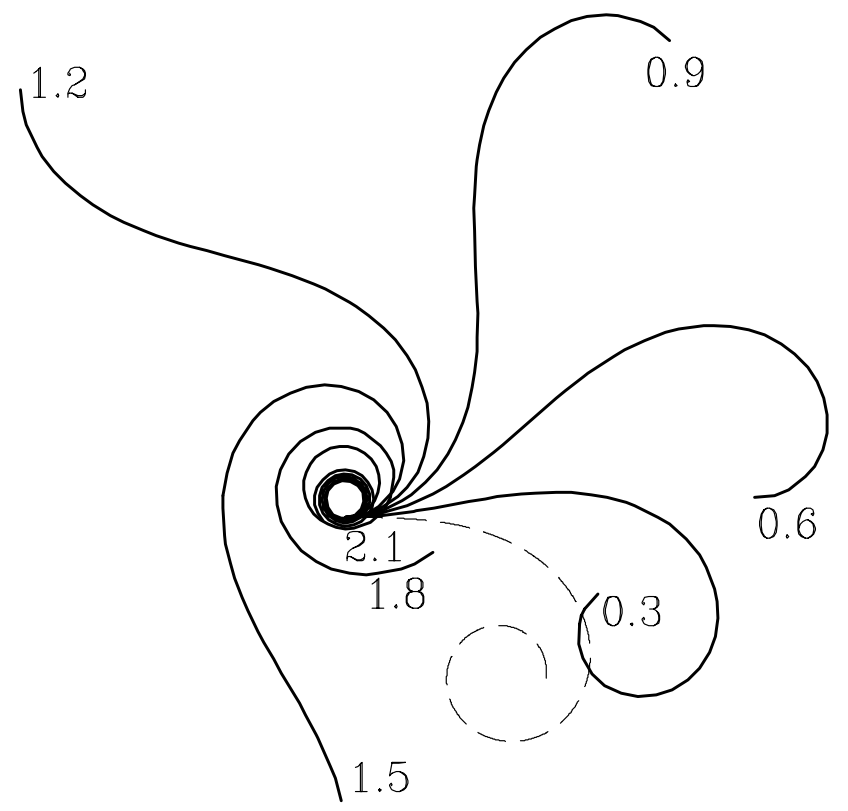

Saje, Turk, Kalagasidu, Vratanar, Figure 13. 


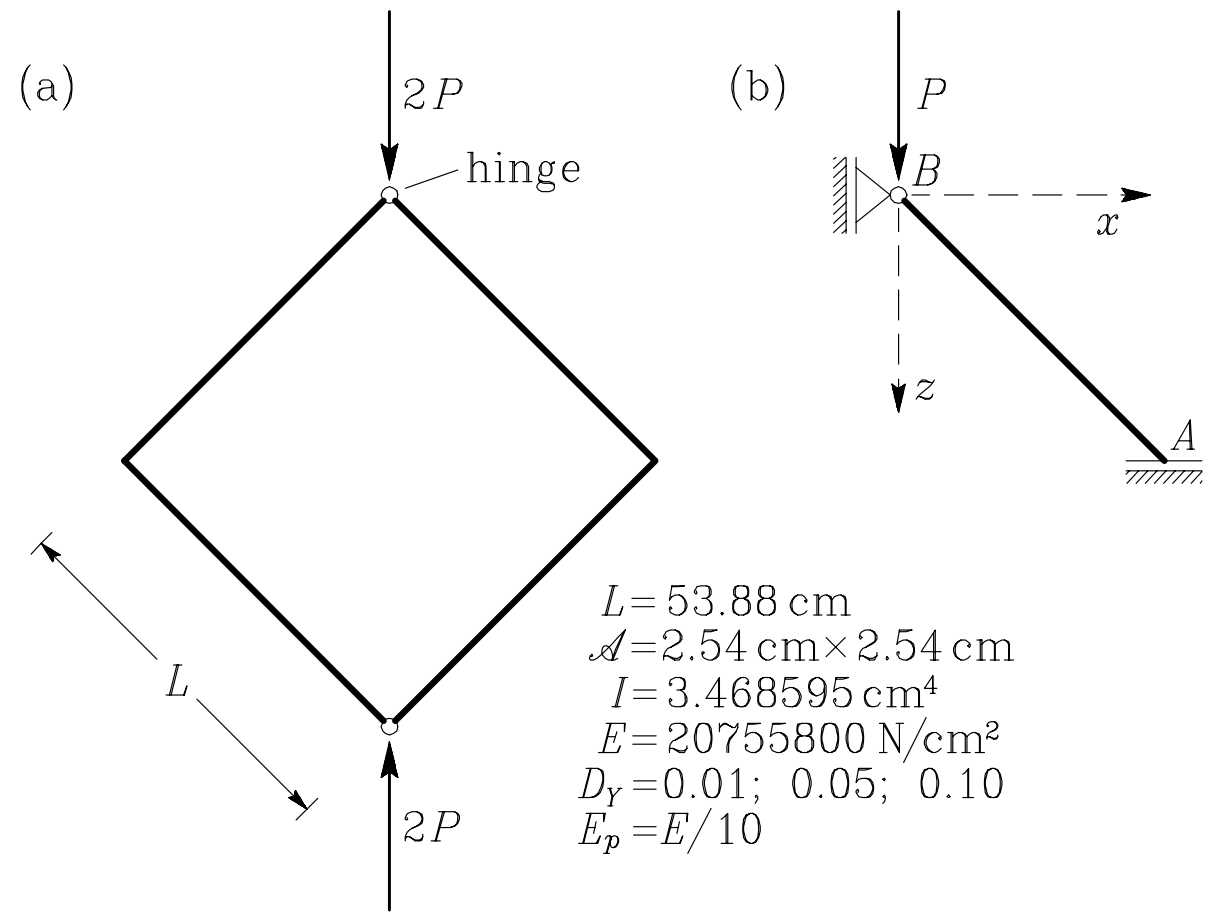

Saje, Turk, Kalagasidu, Vratanar, Figure 14. 


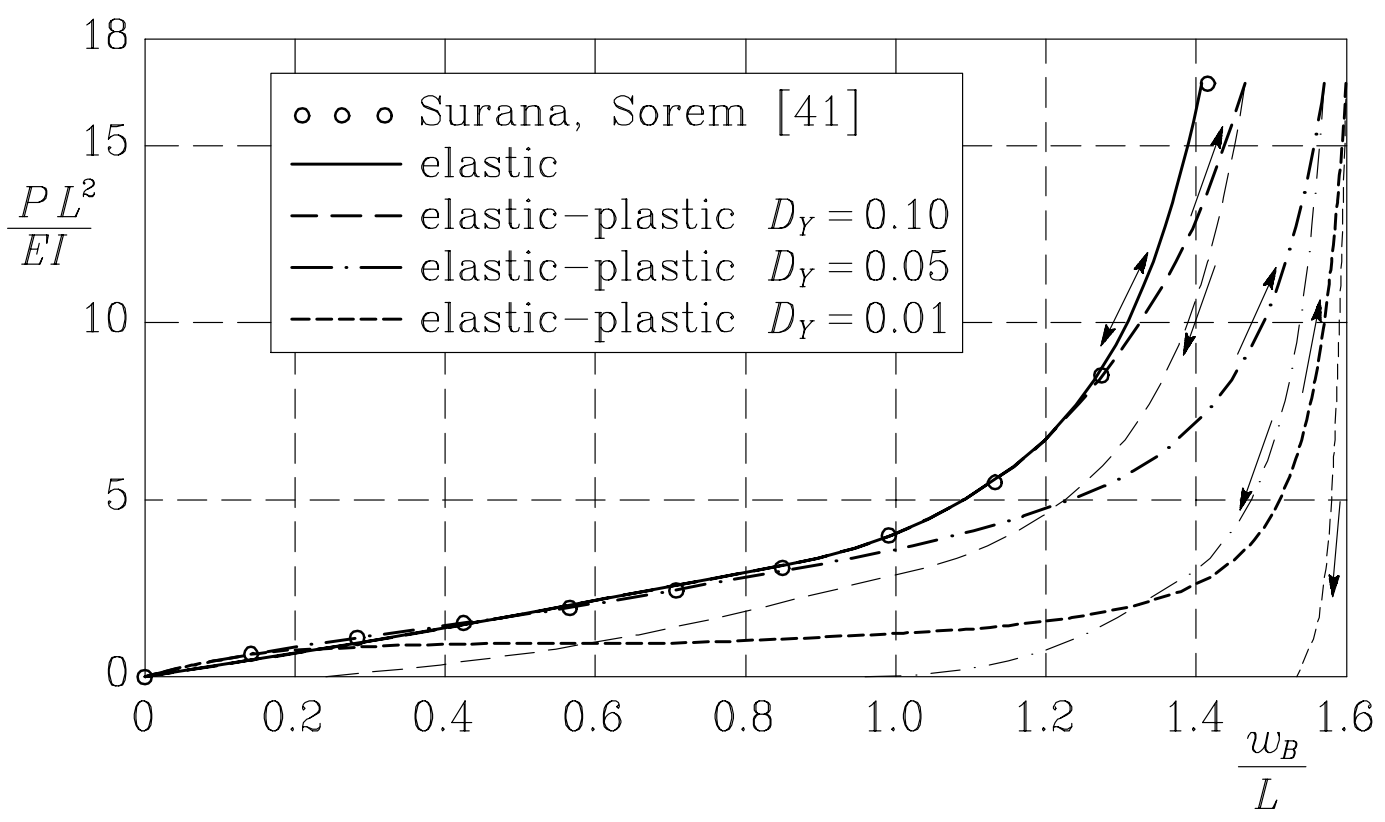

Saje, Turk, Kalagasidu, Vratanar, Figure 15. 
Figure 1: A beam element. Global and material coordinate systems.

Figure 2: Interpolation points of a finite beam element.

Figure 3: A shalow circular arch.

Figure 4: A deep circular arch.

Figure 5: A circular ring subjected to a cyclic force $P$.

Figure 6: A circular ring. Load-deflection relationship.

Figure 7: Deformed shapes of a circular ring for $P=1.779 f \mathrm{kN}(f=0, \pm 1, \pm 2, \ldots, \pm 10)$.

Figure 8: Variation of $1 / C_{11}$ and $C_{22}-C_{12}^{2} / C_{11}$ along the quarter of the ring at $P=17.79 \mathrm{kN}$.

Figure 9: Finite element model of a complete circular ring.

Figure 10: A clothoid shaped spring.

Figure 11: A clothoid shaped spring. Moment - tip rotation curve.

Figure 12: A clothoid shaped spring. Moment - tip displacements curve.

Figure 13: Deformed shapes of a clothoid shaped spring.

Figure 14: A diamond shaped frame and a computational model.

Figure 15: A diamond shaped frame. Force - tip deflection curve. 